Artículo de investigación

\title{
ANATOMÍA XILEMATICA DE CAESALPINIACEAE REGISTRADAS EN LA XILOTECA DE LA UNIVERSIDAD DISTRITAL FRANCISCO JOSÉ DE CALDAS
}

\author{
Xylem anatomy of the Caesalpiniaceae registered in the wood collection of the \\ Universidad Distrital Francisco José de Caldas
}

Palabras clave: Caesalpiniaceae, madera, biometría, características macroscópicas y microscópicas.

Key words: Caesalpiniaceae, wood, biometric, macroscopic and microscopic characteristics

\author{
Esperanza N. Pulido Rodríguez ${ }^{1}$ \\ Durley Mateus ${ }^{2}$ \\ Ivan Lozano $D^{3}$
}

\section{RESUMEN}

El estudio anatómico del xilema de 21 especies de la familia Caesalpiniaceae registradas en la xiloteca José Anatolio Lastra Rivera (JALR) de la Universidad Distrital Francisco José de Caldas, comprendió la caracterización macroscópica, microscópica y biométrica de los elementos celulares que componen el tejido xilemático. Estos análisis se realizaron de acuerdo con los parámetros definidos por el comité de la International Association Wood Anatomist (IAWA Committee 1989) y con los métodos del Laboratorio de Tecnología de Maderas de la Universidad Distrital. Con los resultados obtenidos se elaboraron claves de identificación y análisis de similitud. Las especies estudiadas presentaron características comunes en la diferenciación de anillos de crecimiento, porosidad difusa, presencia de poros solitarios, con gomas, diámetros grandes y segmentos vasculares de longitud corta a mediana; vasos con platinas de perforación simple y punteaduras intervasculares alternas ornadas; parénquima axial paratraqueal aliforme, vasicéntrico, en bandas y presencia de cristales prismáticos. Igualmente, se encontraron variaciones en características anatómicas diagnosticas para algunos géneros como Copaifera que presentó ca- nales gomíferos longitudinales. La variación y el análisis de las características anatómicas del tejido xilemático de estas especies permitió comprobar relaciones taxonómicas de la familia como sucedió con Mora megistosperma, Mora oleífera, Peltogyne pubescens, Peltogyne paniculata, Sclerolobium odoratissimum y Tachigali polyphylla.

\begin{abstract}
The anatomical study of the xylem of 21 species of Caesalpiniaceae registered in the wood collection "José Anatolio Lastra Rivera" (JALR), of the "Universidad Distrital Francisco José de Caldas", included the macroscopic, microscopic and biometric characterization of the cellular elements that constitute the xylematic tissue. These analyses were developed following parameters defined by the International Association Wood Anatomist Committee (IAWA Committee 1989) and methods established by the Wood Technology Laboratory of Universidad Distrital. Measurements and descriptions were used to prepare identification keys and similarity analysis. The wood of Caesalpiniaceae family illustrated common characteristics in growth rings differentiation, porosity, vessel arran-
\end{abstract}

1 Laboratorio de Tecnología de Maderas. Universidad Distrital Francisco José de Caldas. enpulidor@udistrital.edu.co. Autora para correspondencia

2 Universidad Distrital Francisco José de Caldas. Auxiliar de Investigación.

3 Universidad Distrital Francisco José de Caldas. Auxiliar de investigación. 
gement, deposits, diameter and length, plates perforation type, alternate and vestured intervessel pits; fibers wall thickness and length; paratracheal axial vasicentric parenchyma, aliform and banded parenchyma and presence of prismatic crystals. Also, variations in anatomical features such us longitudinal channels were found as diagnostic for some genera like Copaifera. The variation and analysis of anatomical characteristics of the xylem tissue allowed to verify some taxonomic relations of the family Caesalpinaceae, like the observed with the species Mora megistosperma, Mora oleifera, Peltogyne pubescens, Peltogyne paniculata, Sclerolobium odoratissimum and Tachigali polyphylla .

\section{INTRODUCCION}

De acuerdo con el sistema de clasificación filogenética APG II, la familia Fabaceae está integrada por tres subfamilias: Mimosoideae, Caesalpinioideae y Faboideae (Stevens 2001; Chase 2003). Para efectos de la presente investigación, se tuvo en cuenta el sistema de clasificación de plantas con flor desarrollado por Cronquist (1981), en el cual se otorga la categoría de familia a Caesalpiniaceae, Fabaceae y Mimosaceae dentro del orden Fabales.

Caesalpiniaceae es una familia botánica con distribución cosmopolita, que comprende alrededor de 150 géneros y 2200 especies de hierbas, bejucos, arbustos y árboles. En América tropical se encuentran alrededor de 500 árboles de importancia por su uso como ornamental, sombrío y para obtención forraje y madera (Mahecha \&Echeverri 1983). Así mismo, presenta un amplio rango de distribución geográfica en Colombia, localizándose en forma natural en zonas tropicales secas, húmedas y muy húmedas en las regiones Caribe, Pacifica, en los valles interandinos del Cauca y Magdalena, en la Amazonia y parte de la Orinoquia (López \& Montero 2005; Cárdenas \& Salinas 2007).

Díaz (1963), Kribs (1968), JUNAC (1981), Detienne \& Jacquet (1983), Soerianegara \& Lemmens (1993), Richter \& Dallwitz (2000), León \&Espinoza (2001), Miller \& Detienne (2001) y Moya et al (2007) estudiaron la anatomía de la ma- dera de Caesalpiniaceae, principalmente a nivel de especies pero a su vez se ocupan de otros grupos taxonómicos por lo cual no se pueden establecer relaciones anatómicas en los diferentes niveles jerárquicos de esta familia.

Quirk (1983), Nardi \& Edlmann (1992), Gassen (1999) y Höhn (1999), estudiaron la anatomía del xilema de leguminosas de importancia en Asia, África, Australia e Italia, mientras que Espinoza \& Melandri (2006) investigaron sobre la estructura anatómica de la madera de la tribu Caesalpinieae, incluyendo los géneros y especies más importantes de las Caesalpiniaceae. Estos autores citan otros tratados de la anatomía de la madera de las tribus, géneros y especies de esta familia con importantes avances en la caracterización anatómica de maderas oriundas de países latinoamericanos como México, Argentina, Venezuela y Bolivia (Cozo 1951, Reinders-Gouwentak 1955, Koeppen 1980 , Baretta-Kuipers1981, Loureiro \& Silva 1981, Loureiro et al.1983, Ranjani \& Krishnamurthy 1988, Barajas-Morales \& León 1989, Angarita 1991, Espinoza de Pernía et al. 1998 y Gasson et al. 2003 cit. en. Espinoza (2006) y Melandri (2009).

En Colombia, los estudios anatómicos del tejido xilemático son escasos, incompletos o desactualizados y para algunas especies no se registra información. En este estudio, se realizaron descripciones anatómicas de algunas de las maderas incluidas en estudios anatómicos de diferentes grupos taxonómicos, como los desarrollados por Barghoorn et al. (1967), PROEXPO (1970), JUNAC (1981), Rodríguez (1988) y Vásquez \& Ramírez (2005).

La familia Caesalpiniaceae abarca especies maderables que ofrecen numerosas posibilidades de uso por sus características estructurales y durabilidad natural. Pero debido a la poca diversificación de los productos del bosque y al desconocimiento de su manejo y conservación, varias de estas especies se encuentran clasificadas dentro de alguna categoría de amenaza. El objetivo del presente trabajo consistió en analizar la estructura anatómica del xilema de este grupo taxonómico, integrando y revalidando los resultados reportados por diferentes 
autores, como aporte al conocimiento de especies que aún no han sido estudiadas en relación con este campo y así aportar nuevos elementos de análisis en investigaciones de carácter filogenético, ecoanatómico y tecnológico.

\section{MATERIALES Y METODOS}

El estudio se realizó en 21 especies de los géneros Cynometra, Dialium, Eperua, Erythrophleum, Heterostemon, Hymenaea, Koompassia, Macrolobium, Mora, Orphanodendron, Peltogyne, Prioria, Sclerolobium y Tachigali; las cuales se encuentran registradas en la Xiloteca José Anatolio Lastra Rivera (JALR) de la Universidad Distrital Francisco José de Caldas de Bogotá (Tabla 1).

De acuerdo con los procedimientos definidos por el Laboratorio de Tecnología de Maderas de la
Universidad Distrital Francisco José de Caldas, de cada una de las muestras de las especies se seleccionaron probetas sin defectos biológicos o físicos, con un tamaño adecuado y en lo posible con presencia de albura y duramen.

El estudio macroscópico se realizó en probetas lijadas en los tres planos de estudio anatómico de la madera. Para la realización del estudio microscópico se extrajeron cubos de madera que fueron sometidos a diferentes métodos de ablandamiento consistentes en inmersión en agua caliente a una temperatura aproximada de $130^{\circ} \mathrm{C}$ bajo presión, durante diferentes periodos de tiempo, dependiendo de la densidad de la madera. Se realizaron cortes directos sobre el tejido xilemático con un micrótomo de rotación. Estos tejidos fueron sometidos a procesos de tinción con fucsina básica (1\%), fijación con ácido pícrico (1\%), lavado con Etanol en

Tabla 1. Nombre científico, registro y procedencia de los individuos estudiados

\begin{tabular}{|c|c|c|c|c|c|}
\hline & NOMBRE CIENTÍFICO & $\begin{array}{c}\text { ID } \\
\text { XILOTECA }\end{array}$ & COLECCIÓN & $\begin{array}{c}\text { ID } \\
\text { SPECIFY }\end{array}$ & PROCEDENCIA \\
\hline 1 & Batesia floribunda Spruce ex Benth. & 912 & PRORADAM & 912 & Caquetá - Colombia \\
\hline 2 & Copaifera officinalis (Jacq.) L. & 507 & PRORADAM & 507 & Pucalpa - Perú \\
\hline 3 & Crudia glaberrima (Steud.) J.F. Macbr. & 18 & USAID & 1378 & Chocó - Colombia \\
\hline 4 & Cynometra martiana (Hayne) Baill. & 829 & PRORADAM & 829 & Rio Orinoco - Colombia \\
\hline 5 & Dialium guianense (Aubl.) Sandwith. & 270 & PRORADAM & 270 & Antioquia-Colombia \\
\hline 6 & Eperua purpurea Benth. & 792 & PRORADAM & 792 & Bajo Inírida- Colombia \\
\hline 7 & Erythrophleum guineense G. Don. & 242 & PRORADAM & 242 & Guinea - Española \\
\hline 8 & Heterostemon vageleri Harms. & 260 & PRORADAM & 260 & Antioquia-Colombia \\
\hline 9 & Hymenaea courbaril L. & 646 & PRORADAM & 646 & Brasil \\
\hline 10 & Hymenaea oblongifolia Huber. & F192 & FERROCARRILES & 1442 & V. Cauca - Colombia \\
\hline 11 & Koompassia malaccensis Maing. & 318 & PRORADAM & 318 & Malasia \\
\hline 12 & Macrolobium angustifolium (Benth.) R.S. Cowan. & 907 & PRORADAM & 907 & Caquetá - Colombia \\
\hline 13 & Mora megistosperma (Pittier) Britton \&Rose. & 401 & PRORADAM & 401 & Pacífico - Colombia \\
\hline 14 & Mora oleifera (Triana ex Hemsl.) Ducke. & 36 & USAID & 1396 & Pacífico - Colombia \\
\hline 15 & Orphanodendron bernalii Barneby \&J.W. Grimes. & 53 & USAID & 1413 & Antioquía-Colombia \\
\hline 16 & Peltogyne paniculata Benth. & 822 & PRORADAM & 822 & Rio Orinoco - Colombia \\
\hline 17 & Peltogyne parvifolia Spruce ex Benth. & 56 & PRORADAM & 56 & Mérida - Venezuela \\
\hline 18 & Peltogyne pubescens Benth. & 148 & PRORADAM & 148 & Santander-Colombia \\
\hline 19 & Prioria copaifera Griseb. & 41 & PRORADAM & 41 & Antioquía-Colombia \\
\hline 20 & Sclerolobium odoratissimum Spruce ex Benth. & 933 & PRORADAM & 933 & Caquetá - Colombia \\
\hline 21 & Tachigali polyphylla Poepp. & 946 & PRORADAM & 946 & Caquetá - Colombia \\
\hline
\end{tabular}


diferentes concentraciones: 25\% 50\% 75\% 95\%, deshidratación con Alcohol, Xilol (50\%, 50\%), y finalmente Xilol (100\%). El montaje y fijación se realizó con bálsamo de Canadá.

Para el estudio biométrico, se llevó a cabo un proceso de disociación celular consistente en la obtención de astillas y cocción de las mismas en una solución de ácido acético glacial y peróxido de hidrogeno de 20 volúmenes (1:2) hasta observar la individualización de los elementos del xilema (García et. al. 2003). La tinción de los elementos disociados se realizó con Safranina (1\%); este producto se mantuvo en medio húmedo.

El análisis macroscópico se realizó sobre las características estructurales, anatómicas y físicas de la madera, teniendo en cuenta los parámetros establecidos por el Laboratorio de Tecnología de Maderas de la Universidad Distrital Francisco José de Caldas y los conceptos desarrollados por León y Espinoza (2001). La descripción microscópica se realizó sobre los elementos prosenquimáticos y parenquimáticos que componen el tejido xilemático teniendo en cuenta los parámetros de maderas duras presentados por IAWA Committe (1989) citado en León \&Espinoza (2001) e Inside Wood (2004).

Los resultados cualitativos del estudio anatómico se presentan en matrices que contienen la carac- terización macroscópica y microscópica de las estructuras anatómicas y su correspondiente valoración; se identifica la presencia de los caracteres distintivos con "punto" (.) y los caracteres dominantes con el signo más $(+)$.

Para la descripción de los caracteres cuantitativos o biométricos, se utilizaron fotomicrografías del tejido xilemático y de muestras de macerado las cuales fueron medidas empleando el software MOTIC Images (2000, ver. 2.1). Los elementos evaluados en el estudio biométrico se relacionan en la tabla 2 .

Con las variables consideradas se realizó un análisis de similitud de especies aplicando el índice de Jaccard, mediante el programa de software PAST, con el cual se procesaron los datos obtenidos en la caracterización y se construyó un gráfico de conglomerados. Para efectuar los análisis se tuvieron en cuenta los siguientes valores de significancia:00.5 para las especies o probetas diferentes; 0.50.65 en donde la similitud de las especies está sujeta al criterio del autor; $0.65-0.8$ para las especies ó probetas similares; y 0.8-1 para las probetas ó especies que son iguales (Figura 8).

A nivel macroscópico, se tomaron dos fotografías correspondientes a los planos longitudinal y transversal de las probetas utilizadas, mientras

Tabla 2. Especificaciones para la medición de las características anatómicas cuantitativas de vasos, fibras y radios.

\begin{tabular}{ccccc}
\hline ELEMENTO & VARIABLE & $\begin{array}{c}\text { No. } \\
\text { MEDICIONES/ ESP. }\end{array}$ & $\begin{array}{c}\text { AUMENTOS MICROSCOPICOS } \\
\text { (Ax DEL OBJETIVO) }\end{array}$ & AUMENTO CAMARA \\
\hline \multirow{3}{*}{ VASOS } & Diámetro & 25 & $10 \mathrm{X}$ \\
& Longitud & 25 & $10 \mathrm{X}$ \\
& Frecuencia & 10 & $5 \mathrm{X}$ \\
FIBRAS & Diámetro Punteaduras & 10 & $100 \mathrm{X}$ & $6 \mathrm{X}$ \\
& Longitud & 25 & $5 \mathrm{X}$ & \\
& Grosor & 3 & $40 \mathrm{X}$ \\
RADIOS & Altura & 25 & $5 \mathrm{X}-10 \mathrm{X}$ \\
& Numero & 10 & $5 \mathrm{X}-10 \mathrm{X}$ \\
\hline
\end{tabular}

Fuente: Adaptación de Richter Dallwitz (2000) 
que a nivel microscópico se tomaron cuatro fotomicrografías, tres de ellas correspondientes a los planos fundamentales de la madera: transversal, radial y tangencial; y otra correspondiente a algunos elementos que fueron disociados para facilitar su medición. Por último, la variación encontrada en las características anatómicas permitió elaborar claves de identificación macroscópica y microscópica a nivel de especie para la familia Caesalpiniaceae.

\section{RESULTADOS}

Las características macroscópicas de las especies objeto de estudio se relacionan en las tablas 3 a 5 , y su caracterización microscópica se describe en las tablas 6 a 10. Adicionalmente, en la tabla 11 se indican los valores cuantitativos obtenidos en el estudio biométrico, registrando el rango dentro del cual se encuentra el promedio de las medidas así como los valores máximo y mínimo.

En las figuras 1 a 7, se presentan las fotografías y fotomicrografías de las probetas y laminillas estudiadas, en las cuales se muestran las principales características anatómicas descritas.

\section{DISCUSIÓN}

Los especímenes estudiados presentaron rasgos anatómicos similares que permiten diferenciarlos de otros grupos taxonómicos. Igualmente, presentan características particulares que las distinguen dentro del grupo de especies de la familia Caesalpiniaceae. A continuación se presenta un análisis tanto de las características comunes, como de las que establecen un grado de diferencia dentro de este grupo taxonómico.

\section{DESCRIPCIÓN ANATÓMICA}

E1 81 por ciento de las especies presentaron anillos de crecimiento delimitados por líneas concéntricas de parénquima marginal, como se pueden observar en C. officinalis, E. purpurea, $H$. vageleri, $H$. courbaril e $H$. oblongifolia. La especie C. martiana presenta anillos de crecimiento definidos por bandas de parénquima asociadas a banda de madera tardía, confirmando parte de la información reportada por Melandri \& Espinoza (2009); sin embargo, estos anillos presentan dificultad para la diferenciación. Igualmente, se observan anillos de crecimiento definidos por la ausencia de poros en una zona angosta de la madera tardía en C. glaberrima, K. malaccensis y $P$. pubescens, por disposición más compacta de las bandas de parénquima en la madera tardía en $D$. guianense y por banda de madera tardía de coloración más oscura en $T$. polyphylla confirmando la información registrada por Espinoza Melandri (2006). Las especies en donde no se diferenciaron los anillos de crecimiento fueron B. \& floribun$d a$, ratificando lo descrito por Detienne \& Jacquet (1983), E. guineense y S. odoratissimum, confirmando la descripción realizada para este género por Mainieri \& Peres (1989).

La presencia de poros medianos y grandes es otro factor común en las especies estudiadas, confirmando la información reportada por Díaz (1963), Junac (1981), Rodríguez (1988), León \& Espinoza (2001),Vásquez \& Ramírez (2005). Sin embargo, las especies del genero Peltogyne presentaron poros pequeños a medianos, ratificando las descripciones anatómicas de este género presentadas por León \& Espinoza (2001) y Miller (2007). La especie $O$. bernalii presenta poros pequeños.

Todas las especies presentaron porosidad difusa, con predominancia de poros solitarios, confirmando lo expuesto por Detienne \& Jacquet (1983), Richter \&Dallwitz (2000), Espinoza \& Melandri (2006), Kribs (1968), León \& Espinoza (2001). Por su parte, las especies E. purpurea, $S$. odoratissimum y T. polyphylla, presentaron poros en patrón diagonal. La presencia de platinas de perforación simple y punteaduras intervasculares areoladas alternas con ornamentos, confirman las descripciones realizadas por los autores que se han ocupado del estudio de las maderas de la familia Caesalpiniaceae, ratificando así que la presencia de ornamentos en las punteaduras intervascula- 

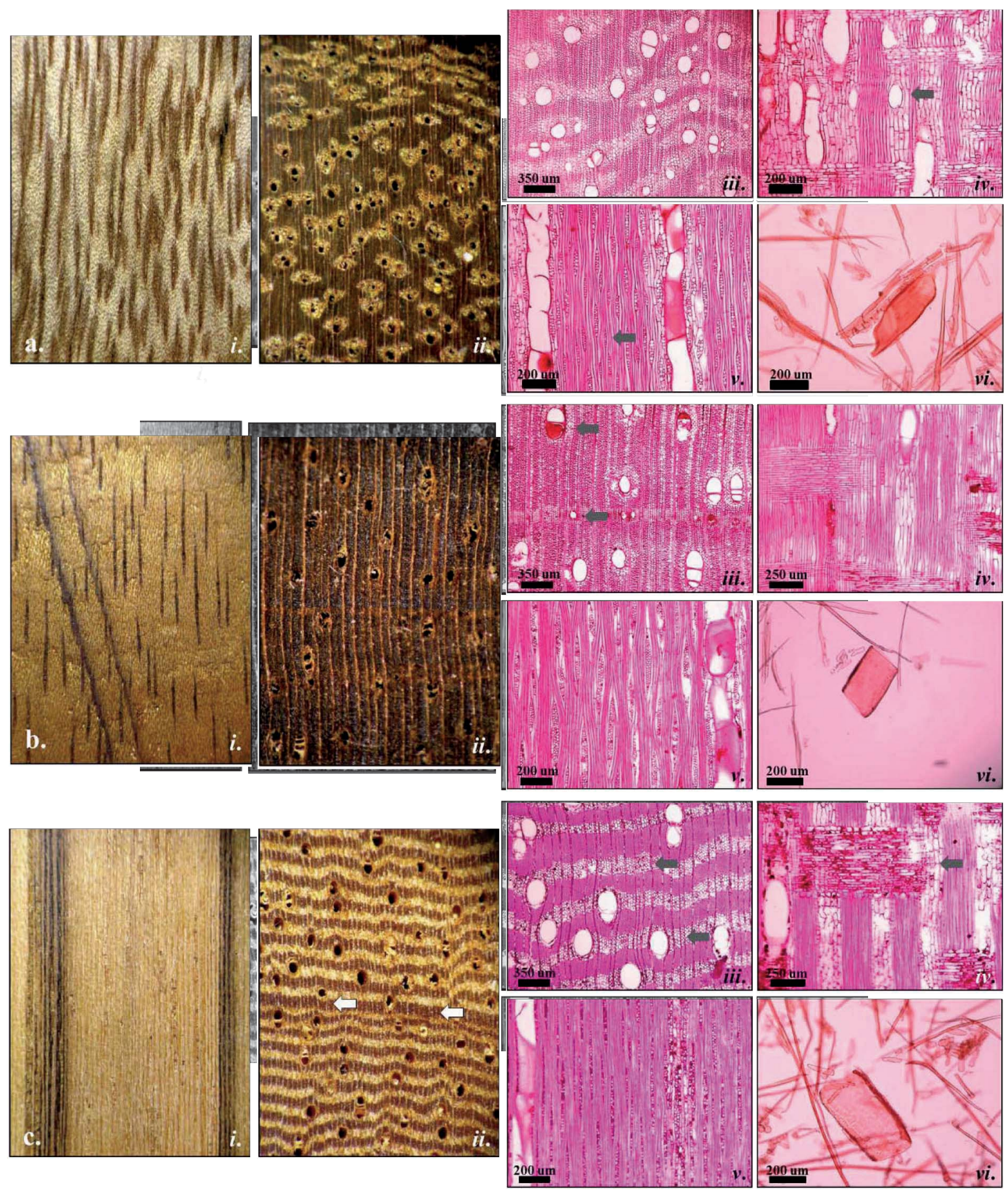

Figura 1. a. Batesia floribunda. Características macroscópicas. i. Plano longitudinal (4x). ii. Plano transversal (10x). Características microscópicas. iii. Plano transversal. iv. Plano radial, células oleíferas asociadas con parénquima axial. v. Plano tangencial, radios bajos. vi. Fibras y elementos disociados. b. Copaifera officinalis. Características macroscópicas. i. Plano longitudinal (4x). ii. Plano transversal (15x). Características microscópicas. iii. Plano transversal, poros grandes con presencia de gomas, canales gomíferos longitudinales. iv. Plano radial. v. Plano tangencial. vi. Fibras y elementos vasculares disociados. c. Crudia glaberrima. Características macroscópicas. $\boldsymbol{i}$ Plano longitudinal (4x). ii. Plano transversal (10x), anillos de crecimiento definidos por ausencia de poros en una zona angosta de la madera. Características microscópicas. iii. Plano transversal, parénquima en bandas anchas. iv. Plano radial, radios Tipo III, con presencia de gomas. v. Plano tangencial. vi. Fibras y elementos vasculares disociados. 

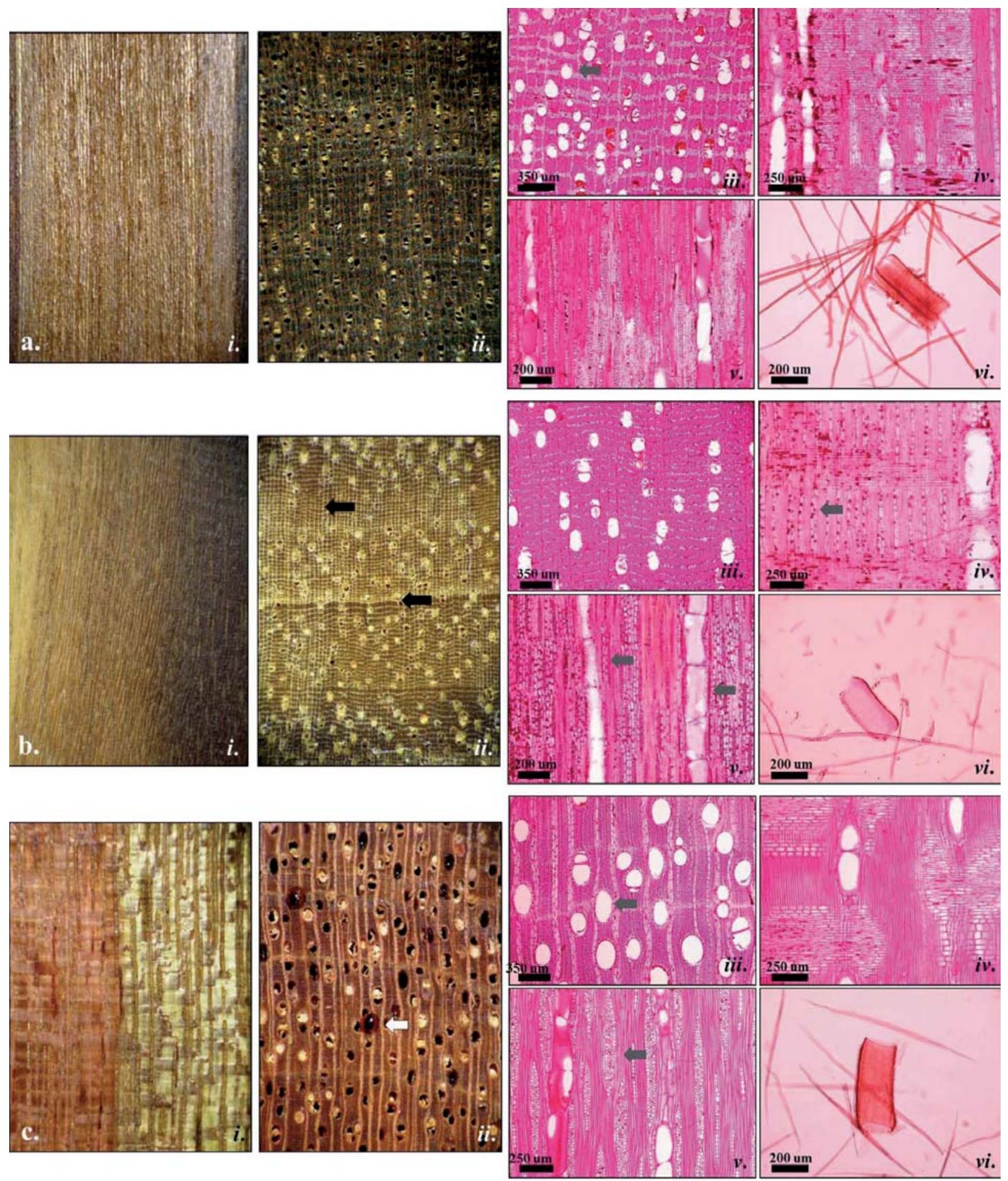

Figura 2. a. Cynometra martiana. Características macroscópicas. i. Plano longitudinal (4x). ii. Plano transversal (10x). Características microscópicas. iii. Plano transversal, poros redondos, poco frecuentes. iv. Plano radial. v. Plano tangencial. vi. Elementos disociados. b. Dialium guianense. Características macroscópicas. i. Plano longitudinal (4x), ii. Plano transversal, parénquima reticulado, anillos de crecimiento definidos por disposición más compacta de las bandas de parénquima en la madera tardía (10x). Características microscópicas. iii. Plano transversal. iv.) Plano radial, cuerpos de sílice presentes en células de parénquima axial. v. Plano tangencial, radios y parénquima estratificados. vi. Elementos disociados. c. Eperua purpurea. Características macroscópicas. i. Plano longitudinal (4x), ii. Plano transversal, poros grandes con abundancia de gomas (10x). Características microscópicas. iii. Plano transversal. iv. Plano radial. v. Plano tangencial, radios altos y multiseriados (4-6 células ancho). vi. Elementos disociados. 

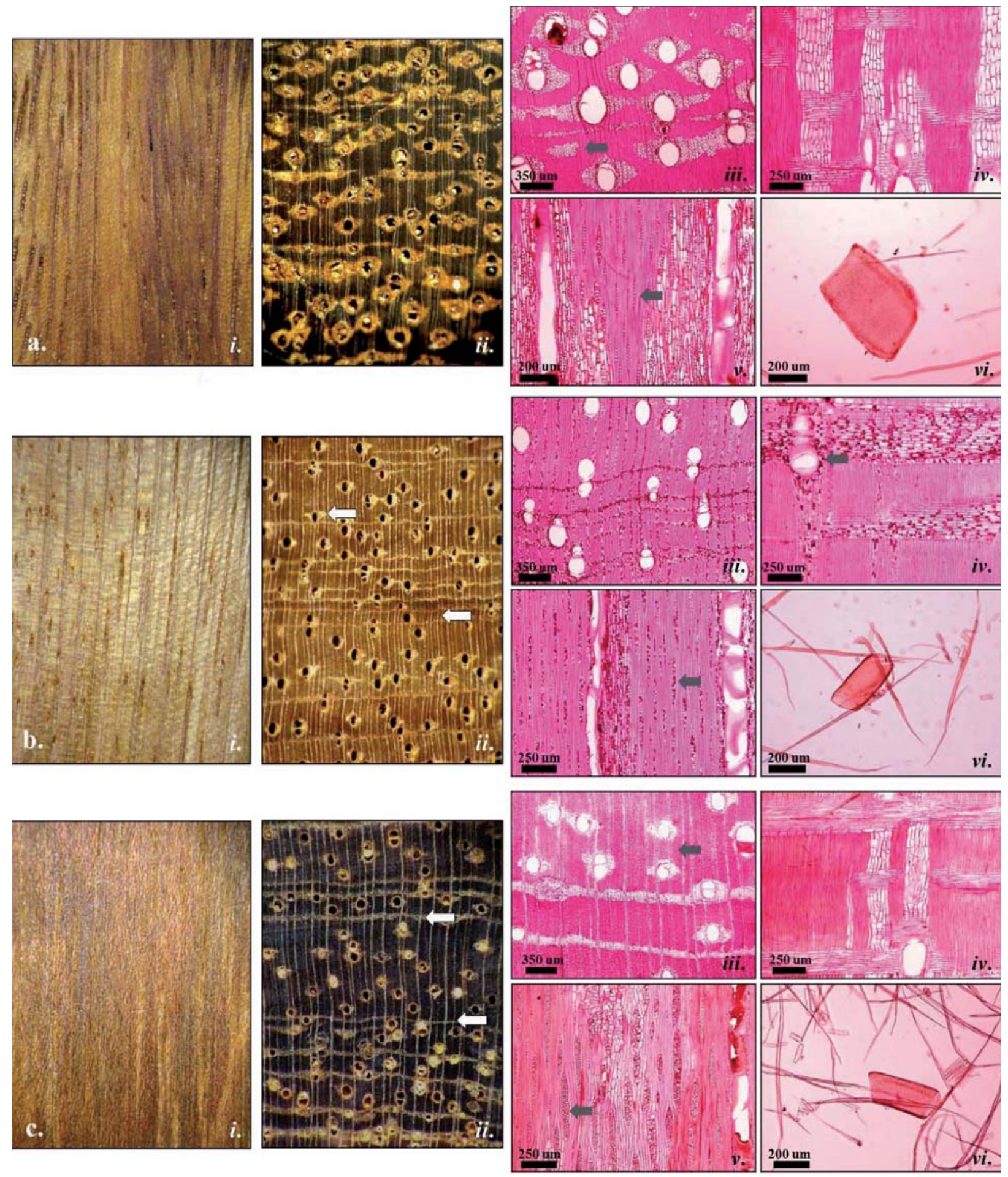

Figura 3. a. Erythrophleum guineense. Características macroscópicas. i. Plano longitudinal (4x). ii. Plano transversal, poros sin patrón de disposición (10x). Características microscópicas. iii. Plano transversal, parénquima apotraqueal difuso en agregados. iv. Plano radial. v. Plano tangencial, radios uniseriados. vi. Elementos disociados. b. Heterostemon vageleri. Características macroscópicas. i. Plano longitudinal (4x). ii. Plano transversal, parénquima aliforme de ala corta y en bandas finas (10x). Características microscópicas. iii. Plano transversal. iv. Plano radial, platina de perforación simple. v. Plano tangencial, radios biseriados. vi. Elementos disociados. c. Hymenaea courbaril. Características macroscópicas. i. Plano longitudinal (4x). ii. Plano transversal, anillos de crecimiento definidos por líneas concéntricas de parénquima (10x). Características microscópicas. iii. Plano transversal, poros muy poco frecuentes. iv. Plano radial. v. Plano tangencial, radios triseriados. vi. Elementos disociados. 

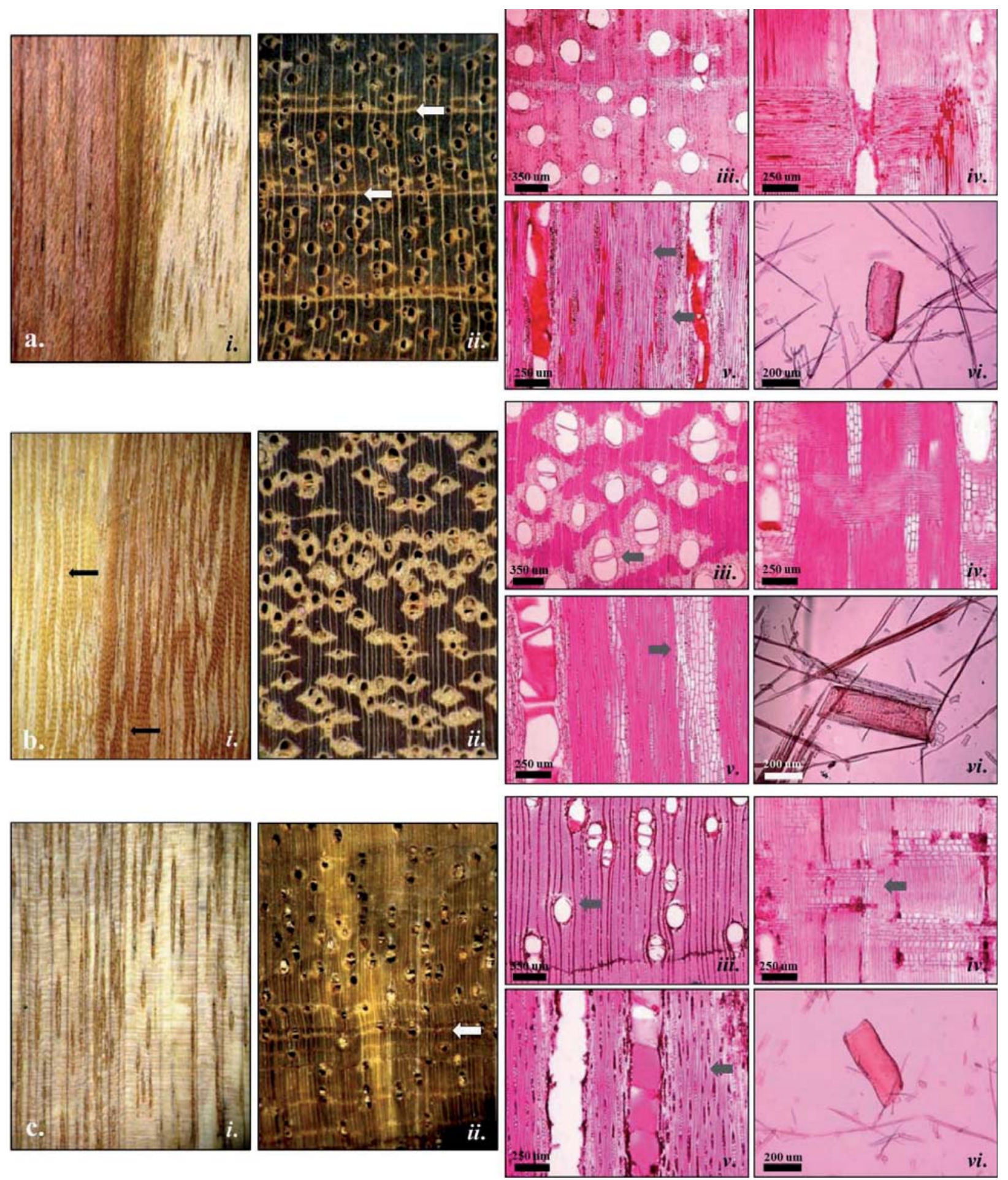

Figura 4. a. Hymenaea oblongifolia. Características macroscópicas. i. Plano longitudinal (4x). ii. Plano transversal, anillos de crecimiento definidos por líneas concéntricas de parénquima (10x). Características microscópicas. iii. Plano transversal. iv. Plano radial. v. Plano tangencial, radios de dos tamaños diferentes. vi. Elementos disociados. b. Koompassia malaccensis. Características macroscópicas. i. Plano longitudinal, presencia de rizos (4x). ii. Plano transversal (10x). Características microscópicas. iii. Plano transversal, poros múltiples radiales cortos. iv. Plano radial. v. Plano tangencial, parénquima irregularmente estratificado. vi. Elementos disociados. c. Macrolobium angustifolium. Características macroscópicas. i. Plano longitudinal (4x). ii. Plano transversal, parénquima en bandas finas (10x). Características microscópicas. iii. Plano transversal, parénquima paratraqueal escaso. iv. Plano radial, radios tipo IV. v. Plano tangencial, radios muy numerosos. vi. Elementos disociados. 

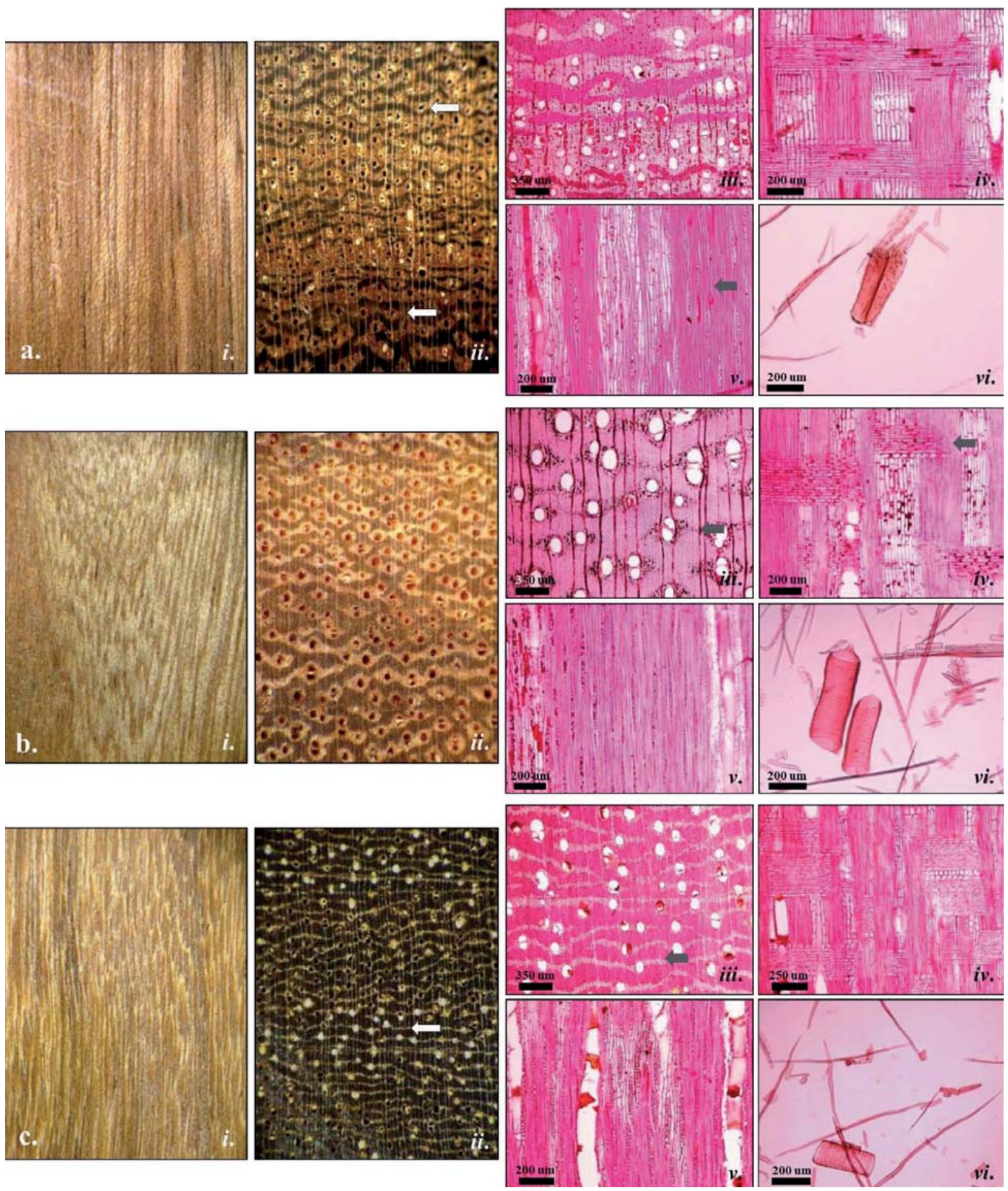

Figura 5. a.Mora megistosperma.Características macroscópicas.i. Plano longitudinal (4x).ii. Plano transversal, parénquima paratraqueal confluente (10x). Características microscópicas. iii.Plano transversal.iv. Plano radial.v. Plano tangencial, radios numerosos.vi. Elementos disociados.b.Mora oleífera. Características macroscópicas. i.Plano longitudinal (4x).ii.) Plano transversal (10x). Características microscópicas. iii.Plano transversal, parénquima paratraqueal aliforme de ala fina y extendida.iv. Plano radial, radios tipo I.v. Plano tangencial.vi. Elementos disociados.c. Orphanodendron bernalii.Características macroscópicas. i. Plano longitudinal (4x).ii. Plano transversal, poros pequeños, con presencia de otro tipo de depósitos (10x). Características microscópicas. iii.Plano transversal, parénquima paratraqueal confluente.iv. Plano radial.v. Plano tangencial.vi. Elementos disociados. 

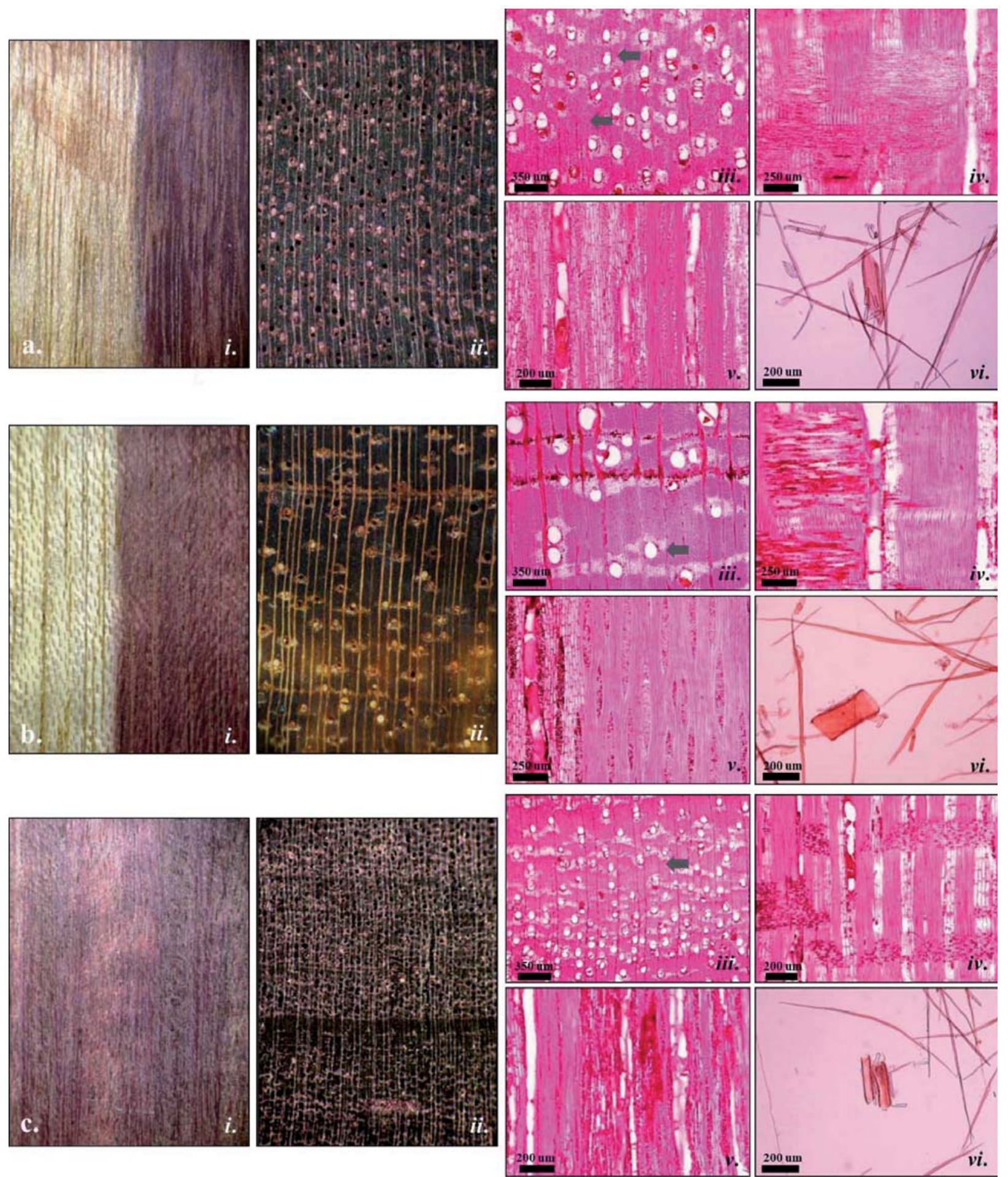

Figura 6. a.Peltogyne paniculata.Características macroscópicas.i. Plano longitudinal (4x).ii. Plano transversal (10x). Características microscópicas. iii.Plano transversal, poros de diámetro mediano, fibras de pared celular gruesa.iv. Plano radial.v. Plano tangencial.vi. Elementos disociados.b.Peltogyne parvifolia.Características macroscópicas. i.Plano longitudinal (4x).ii. Plano transversal (10x). Características microscópicas. iii.Plano transversal, poros predominantemente solitarios.iv. Plano radial.v. Plano tangencial. vi. Elementos disociados.c.Peltogyne pubescens.Características macroscópicas. i. Plano longitudinal (4x).ii. Plano transversal (10x).Características microscópicas. iii.Plano transversal, poros moderadamente frecuentes.iv. Plano radial.v. Plano tangencial.vi. Elementos disociados. 

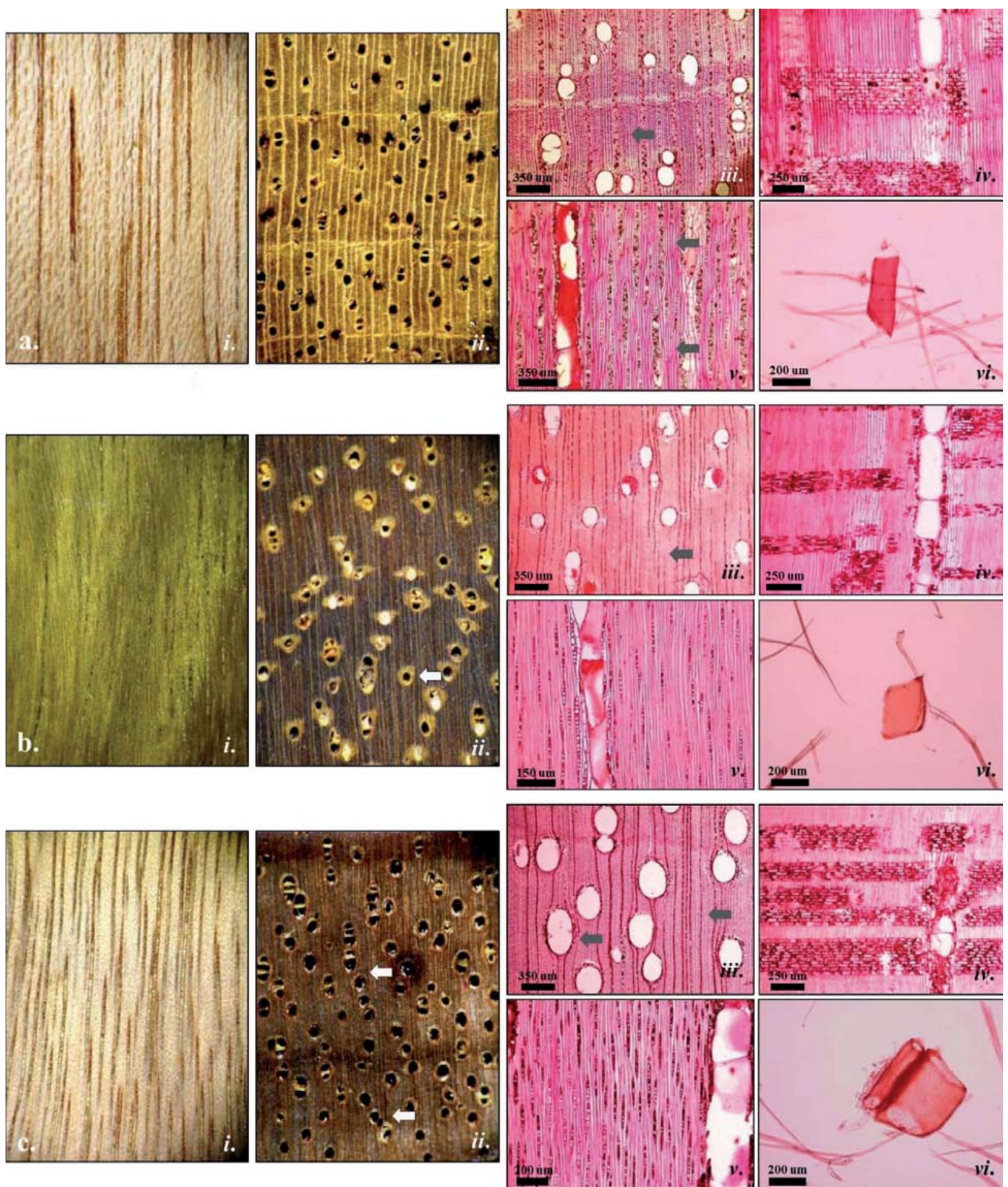

Figura 7. a. Prioria copaifera.Características macroscópicas.i. Plano longitudinal (4x).ii. Plano transversal (10x). Características microscópicas. iii.Plano transversal, fibras de pared celular delgada.iv. Plano radial, iv. Plano tangencial, fibras estratificadas.vi. Elementos disociados.b.Sclerolobium odoratissimum.Características macroscópicas. i. Plano longitudinal (4x).ii. Plano transversal, parénquima paratraqueal vasicéntrico (15x). Características microscópicas. iii. Plano transversal, poros muypocofrecuentes.iv. Plano radial.iv. Plano tangencial.vi. Elementos disociados.c.Tachigali polyphylla.Características macroscópicas. i. Plano longitudinal (4x). ii. Plano transversal, poros conpatrón de disposición diagonal (10x). Características microscópicas. iii.Plano transversal, poros ovalados, fibras de pared celular mediana iv. Plano radial.iv.Plano tangencial.vi. Elementos disociados. 


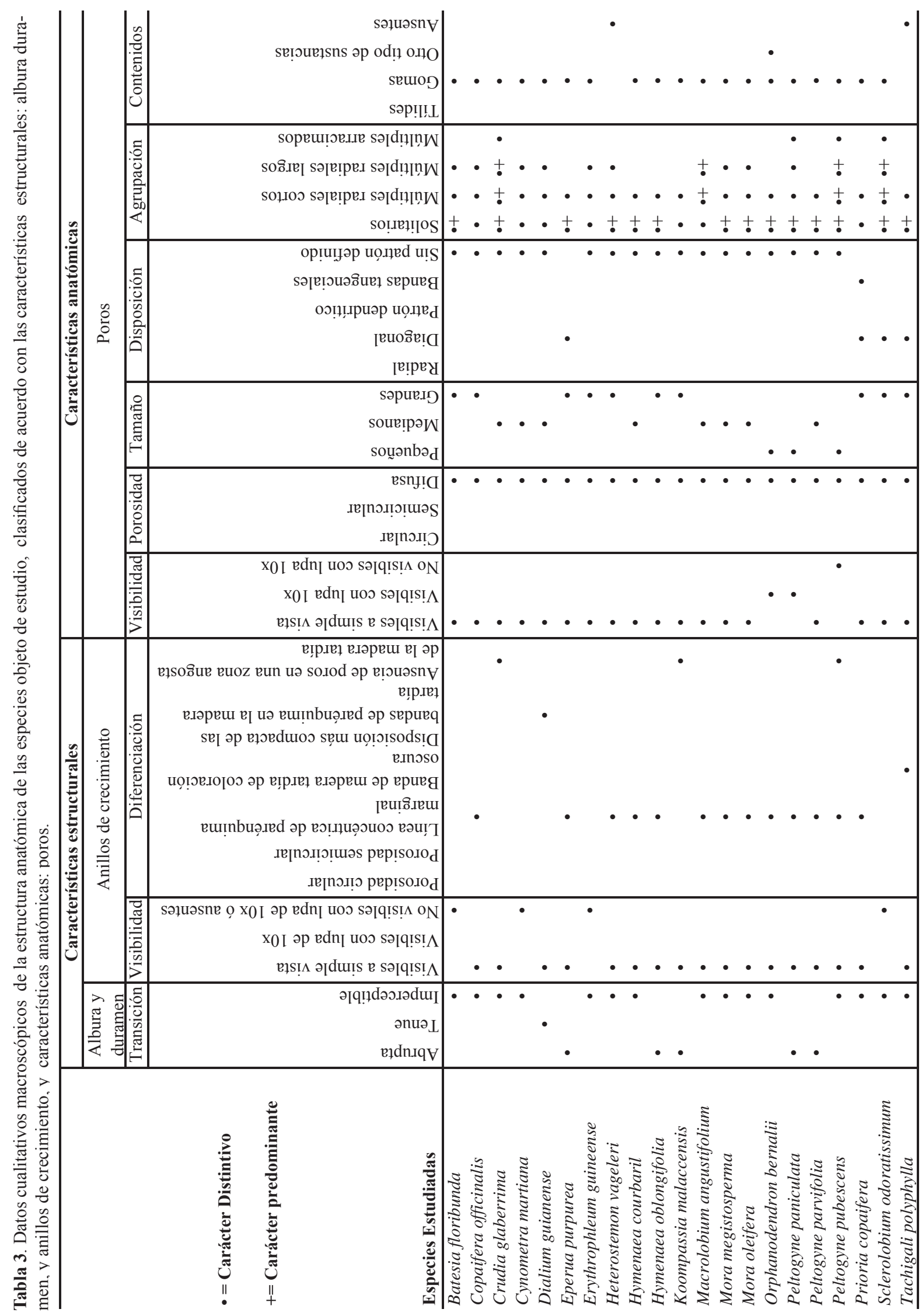




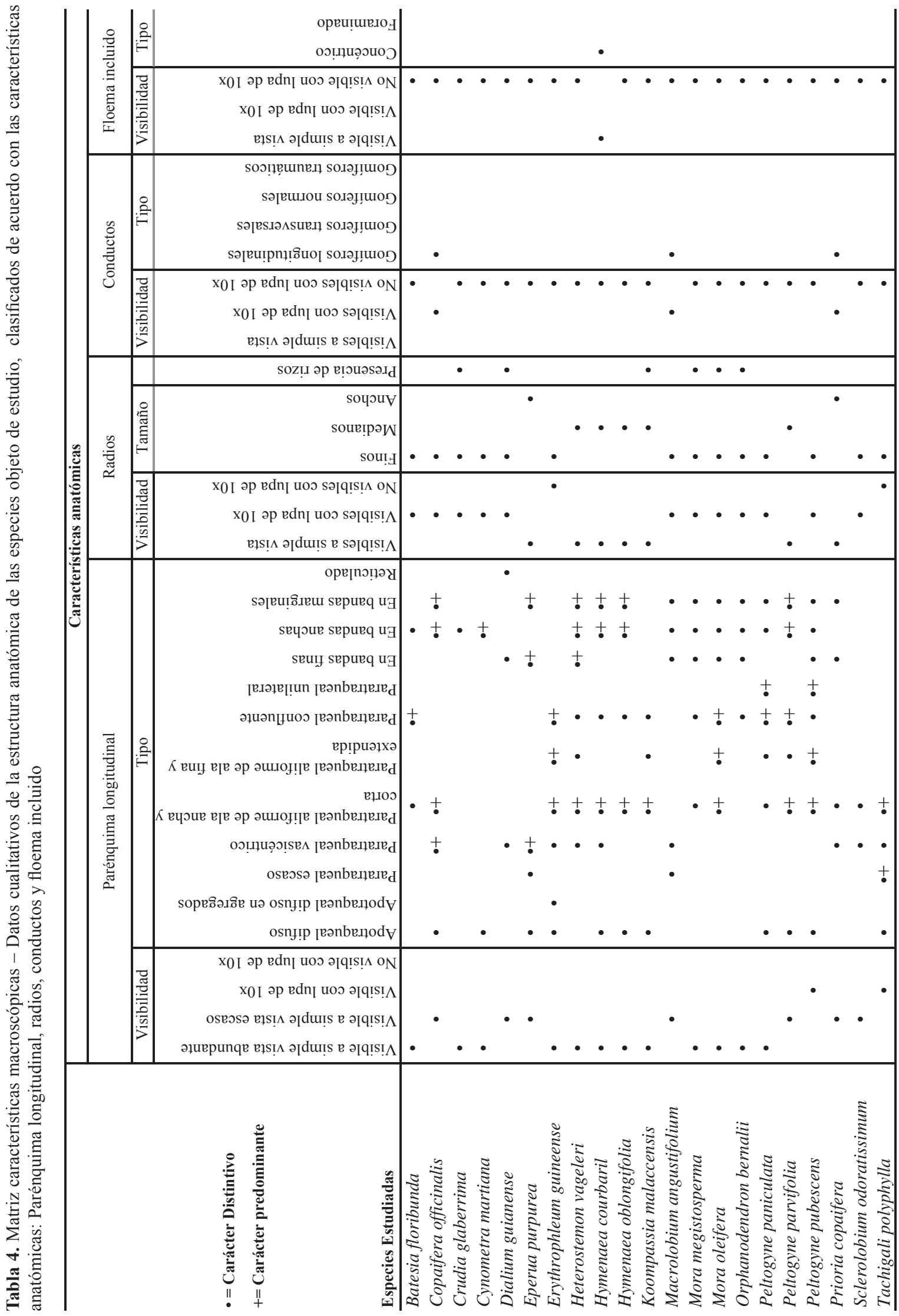




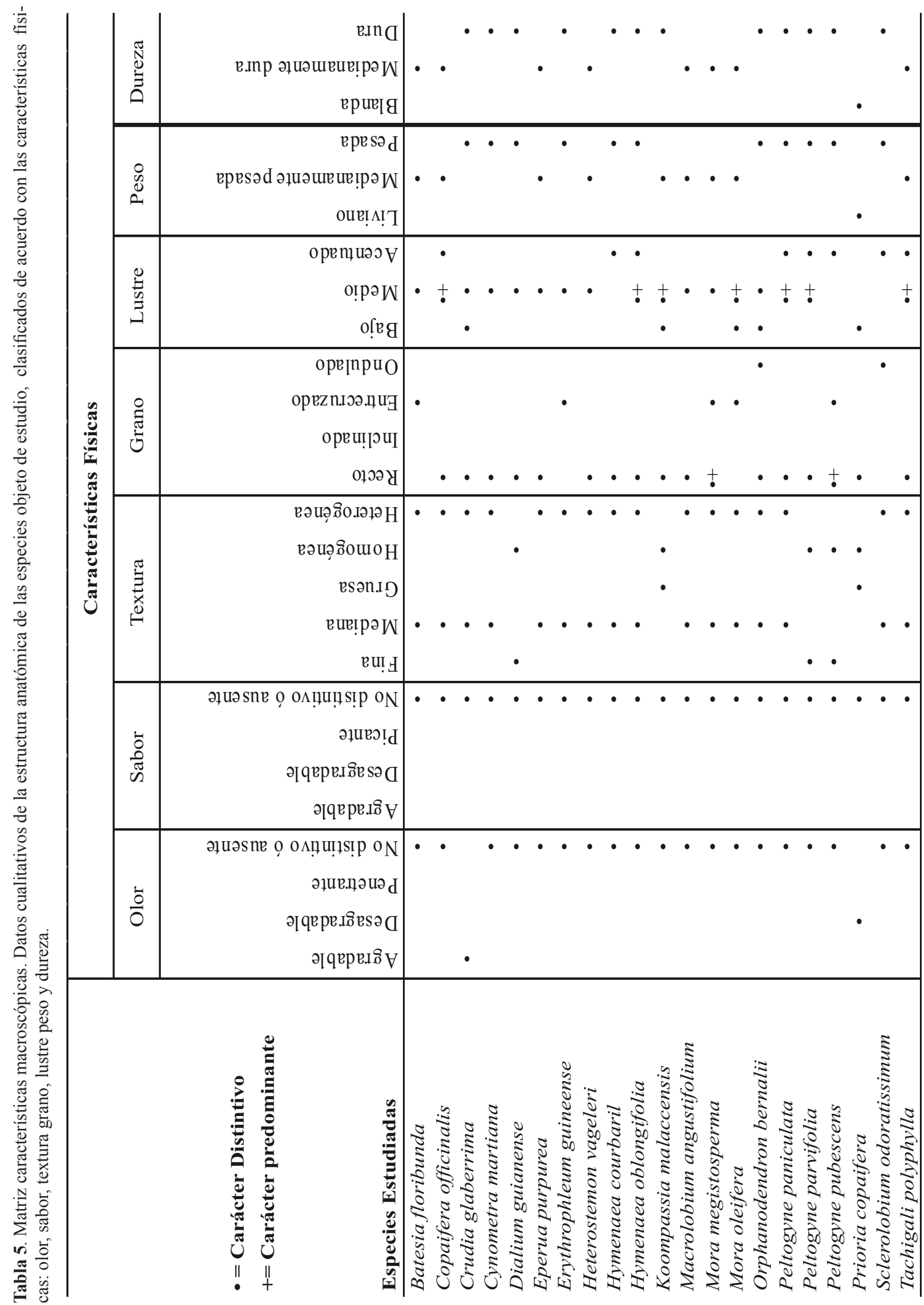




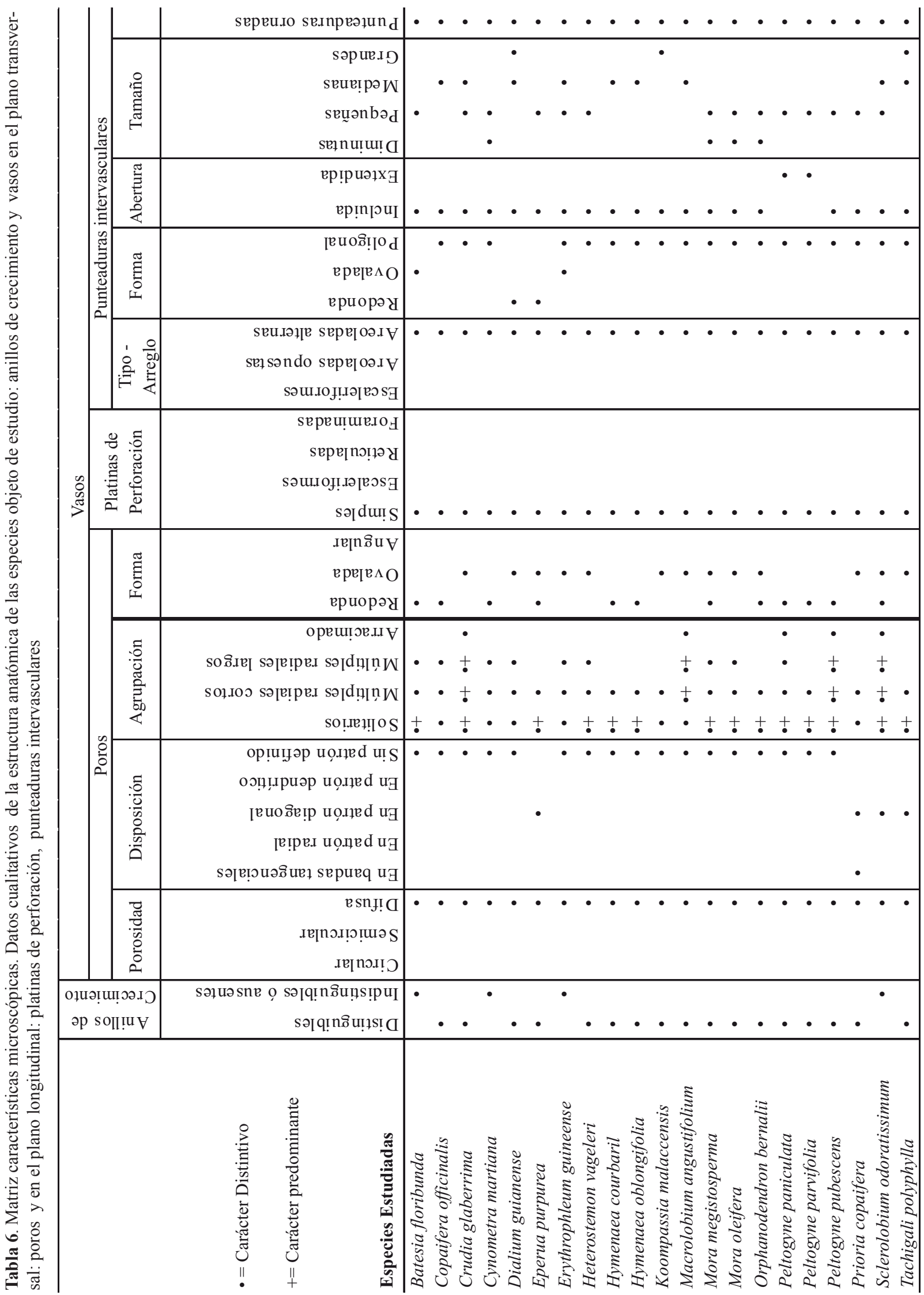




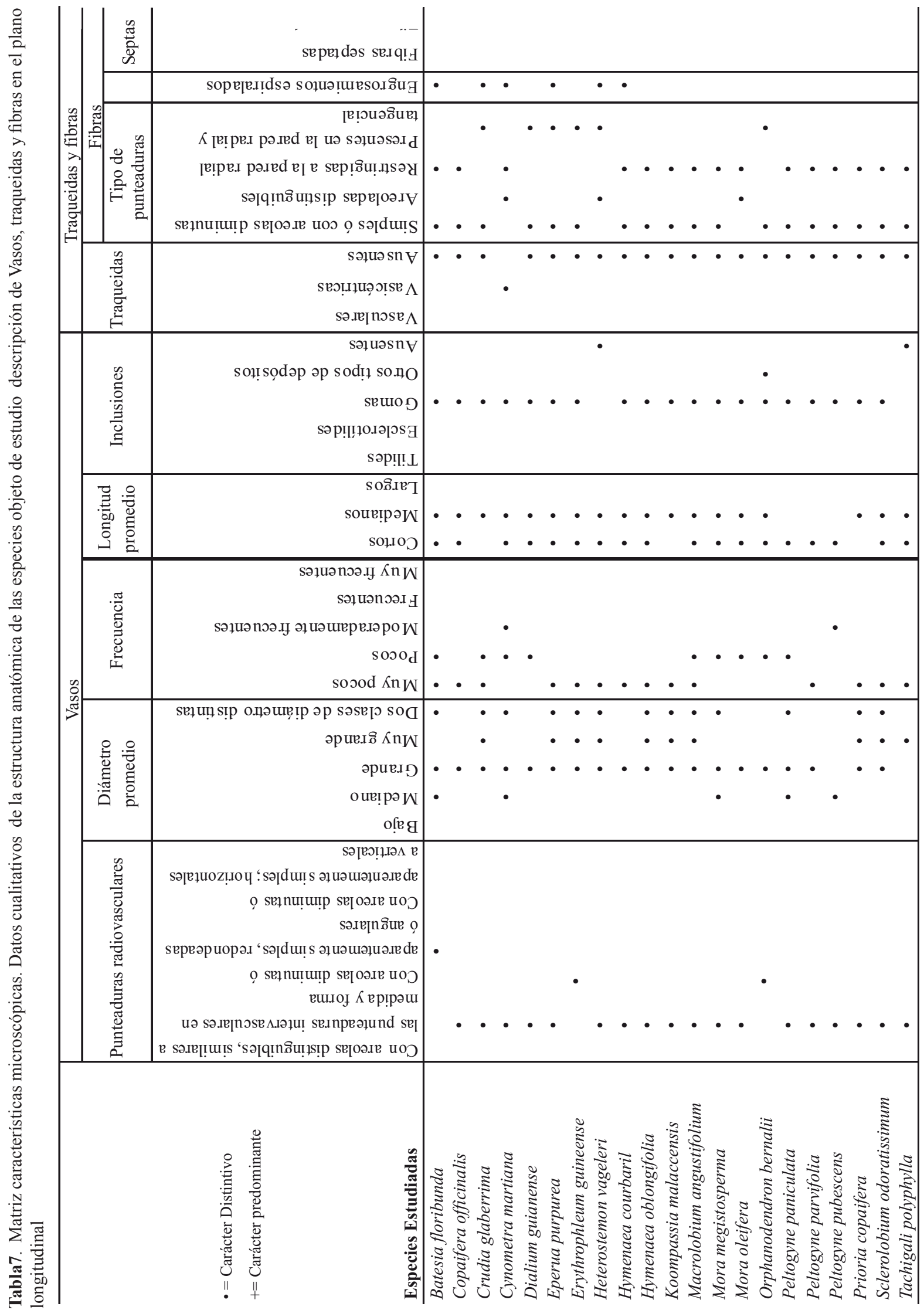




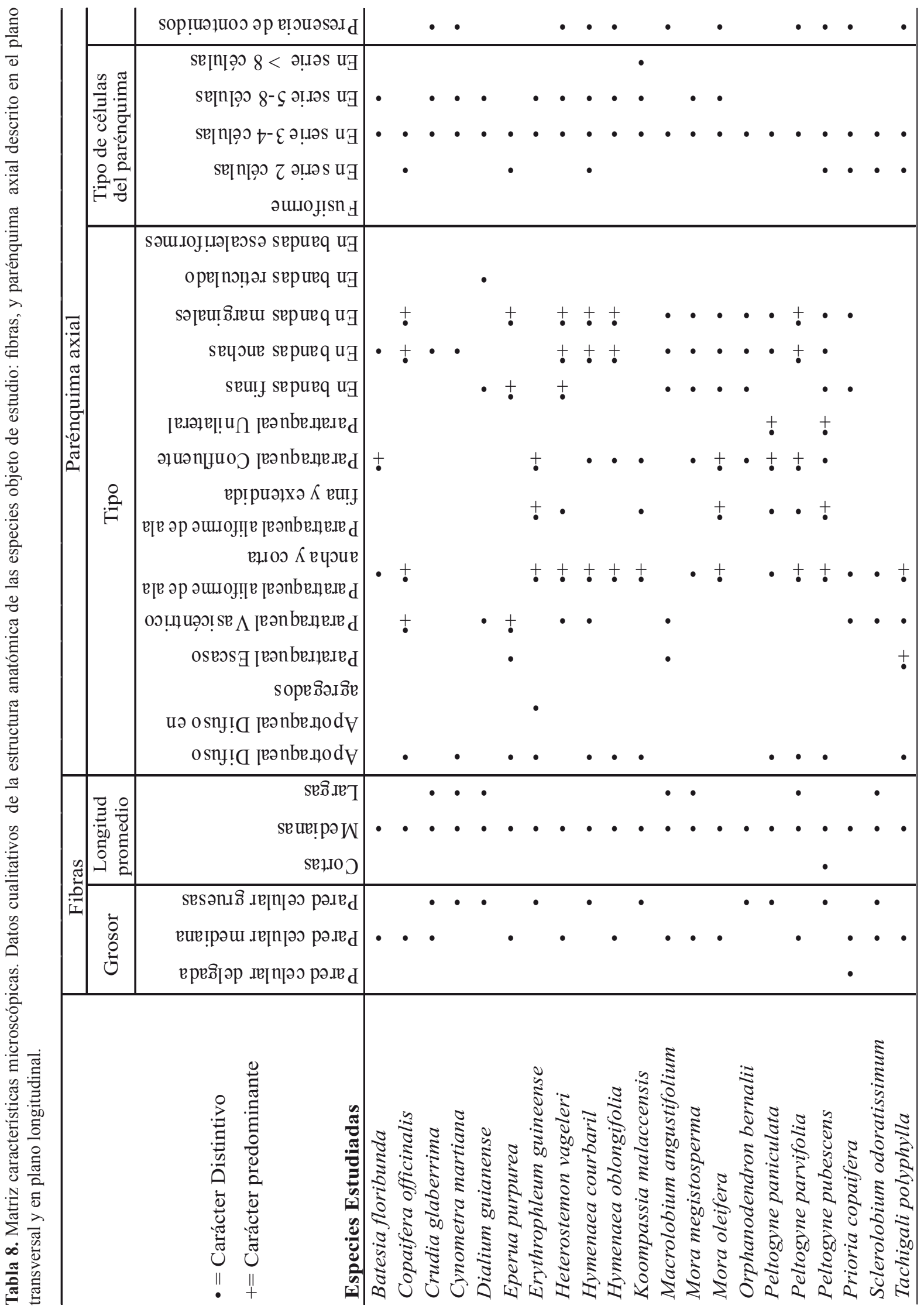




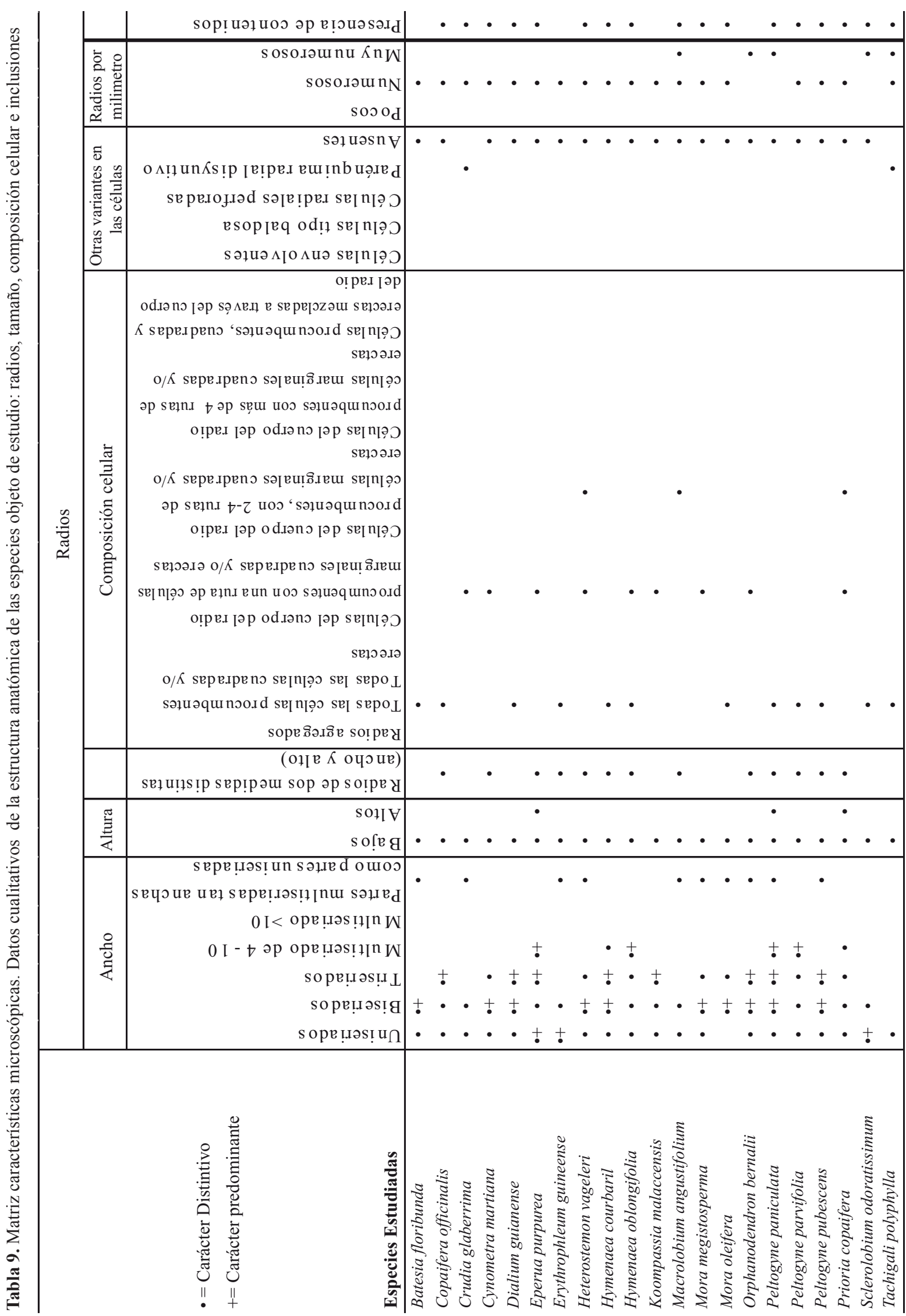




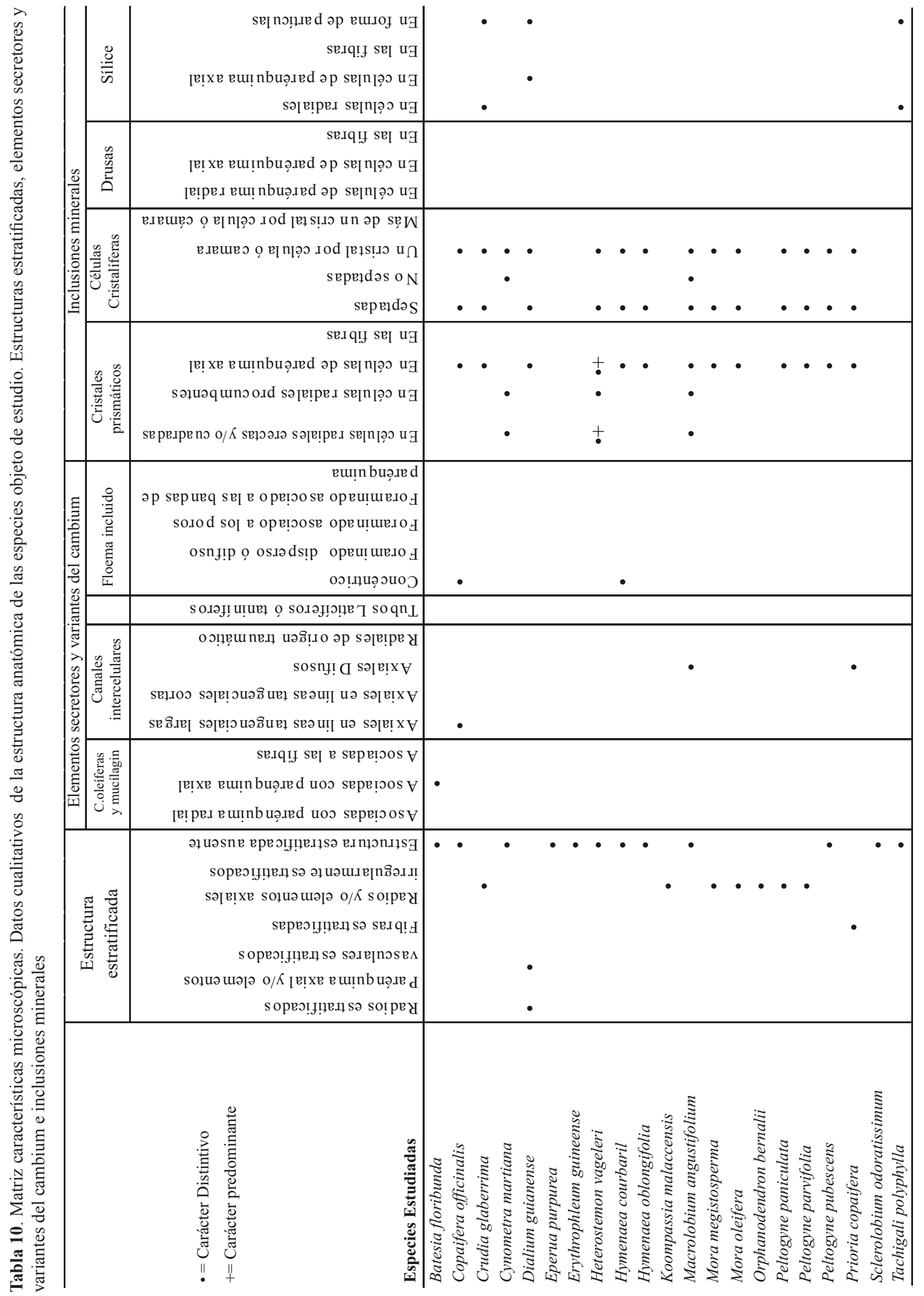


Esperanza N. Pulido Rodríguez, Durley Mateus \& Ivan Lozano D.

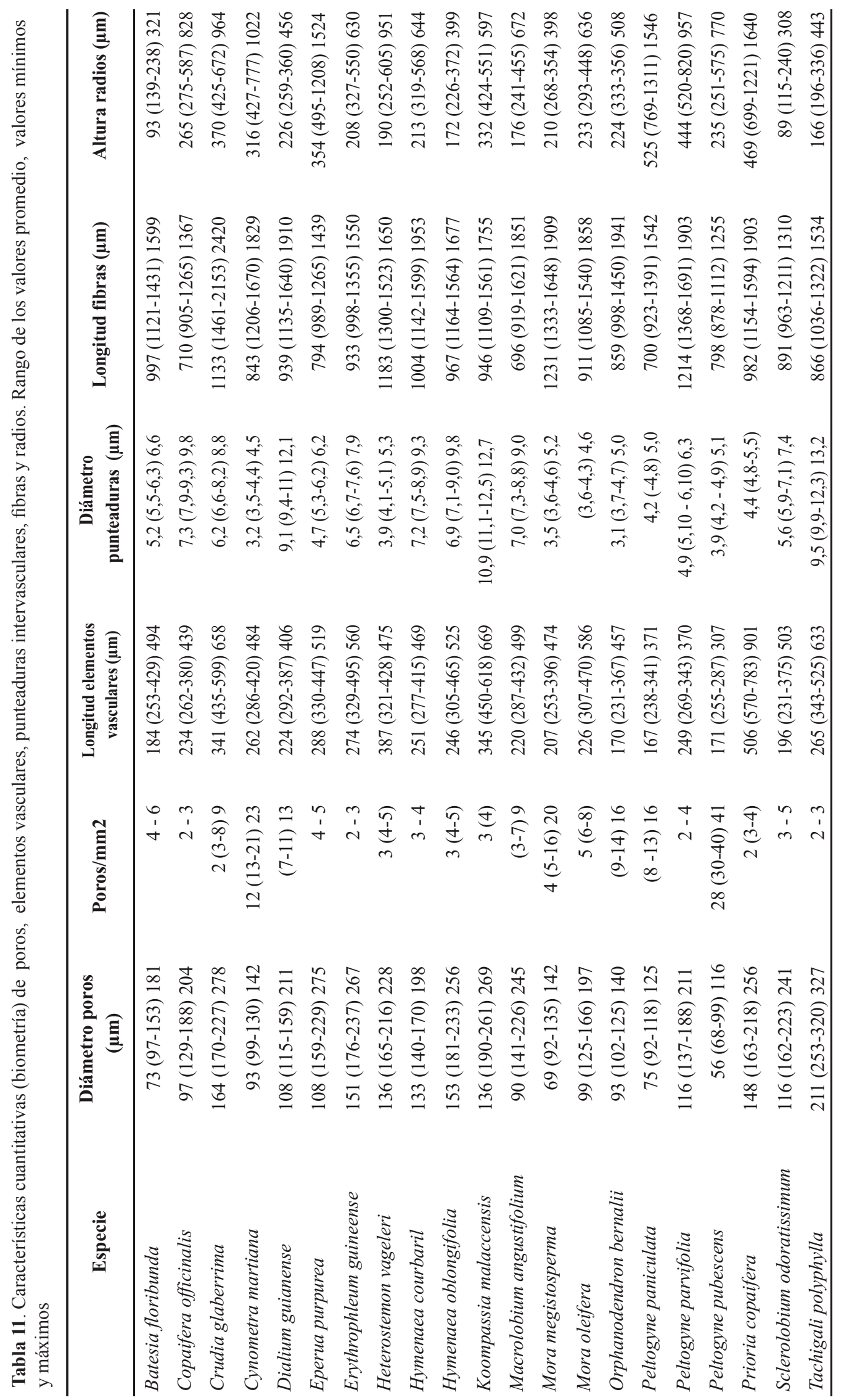


res, desde la sistemática son consideradas como elementos diagnósticos que pueden caracterizar a toda una familia (Carlquist 2001). También se observaron contenidos de tipo gomoso en los poros, siendo éste otro factor común en las especies de estudio ( $90 \%$ de las especies), aunque en O. bernalii se observaron depósitos de tipo cálcico, y H. vageleri y $T$. polyphylla no presentaron contenidos.

Se presentaron fibras no septadas, con una tendencia (85\% de las especies) a desarrollar punteaduras simples o con reborde reducido o fibras libriformes. Al relacionar estos caracteres con las platinas de perforación simples, se confirma uno de los grados de evolución de las estructuras anatómicas del tejido xilemático descrito por Carlquist (2001). Por su parte, las especies C. martia$n a, H$. vageleri y $M$. oleífera contienen fibras con reborde reducido, restringidas a la pared radial y son de pared celular mediana y de pared celular gruesa. Tan sólo P. copaifera presentó fibras de pared celular delgada a mediana, confirmando lo descrito por Detienne \& Jacquet (1983).

Las maderas provenientes de los bosques de zonas bajas tropicales se caracterizan por contener abundante parénquima axial principalmente de tipo paratraqueal (León \& Espinoza 2001); los resultados del presente estudio ratifican esta información. La presencia de parénquima axial apotraqueal, paratraqueal, vasicéntrico, aliforme y en bandas, fue común para la mayoría de las especies. Igualmente, se encontró parénquima en bandas de tipo marginal, en bandas anchas y en menor proporción en bandas finas. Únicamente D. guianense registró parénquima reticulado en bandas confirmando lo descrito por Detienne \& Jacquet (1983) y Höhn (1999). La especie C. martiana, presentó parénquima apotraqueal difuso y en bandas, característica que es común a otras especies de este género confirmando lo descrito por Melandri \& Espinoza (2009).

Las inclusiones minerales se presentaron en forma de cristales prismáticos en células radiales decumbentes en las especies: C.martiana, H. vageleri, y M. angustifolium, mientras que para las especies
C. officinalis, C. glaberrima, D. guianeense, $H$. vageleri, $H$. courbaril, $H$. oblogifolia, M. angustifolium, M. megistosperma, M. oleífera, P. paniculata, P. parvifolia, P. pubescens y P. copaifera, se presentaron cristales prismáticos en células de parénquima axial, en disposición de un cristal por cámara, esta descripción coincide con los análisis presentados por Melandri \& Espinoza (2009). Los cuerpos de sílice se encontraron en las células de parénquima radial en forma de partículas, ratificando los análisis presentados por Koeppen (1980), Baretta-Kuipers (1981), y Gasson et al (2003) citado en Melandri \& Espinoza (2009).

Se presentaron radios leñosos homocelulares y heterocelulares, principalmente uniseriados aunque, también se presentaron biseriados y multiseriados. Las especies, E. guineense, S. odoratissimum y T. polyphylla presentaron radios homocelulares y uniseriados, ratificando lo descrito por Melandri \& Espinoza (2009), las especies B. floribunda, C. officinalis, D. guianense, $H$. courbaril, M. oleífera, P. paniculata, P. pubescens, presentaron radios homocelulares con dos a tres células de ancho y las especies H. courbaril, P. parvifolia presentaron radios homocelulares con más de cuatro células de ancho. Las especies que presentaron radios heterocelulares con células procumbentes y una ruta de células marginales erectas fueron: C. glaberrima, C. americana, C. martiana, E. purpurea, K. malaccensis, M. megistosperma, O. bernalii y $P$. copaifera, mientras que $H$. vageleri, $M$. angustifolium presentaron células procumbentes con dos a cuatro rutas de células marginales erectas confirmando lo descrito por Melandri \& Espinoza (2009). La especie P. copaifera presentó radios uniseriados, radios de dos a tres células de ancho y radios heterocelulares compuestos por células procumbentes con dos a cuatro líneas marginales de células decumbentes. Estas descripciones coinciden con los análisis de las variaciones radiales en especies de la familia Caesalpiniaceae realizados por Melandri \&Espinoza (2006, 2008), 2009), en donde el ancho de los radios son considerados como carácter diagnóstico, siendo frecuente la presencia de radios uniseriados y radios con una a tres células de ancho. Se reconocieron radios 
multiseriados en las especies: C. officinalis, E. purpurea, H. courbaril, H. oblongifolia, P. pubescens, $P$. parvifolia P. paniculata, M. oleífera y $M$. megistosperma, radios multiseriados, coincidiendo con lo descrito por estos mismos autores. Por último, es de mencionar que tan sólo C. glaberrima y $T$. polyphylla presentaron parénquima radial disyuntivo. Los radios presentaron contenidos.

Se encontraron elementos axiales irregularmente estratificados. La especie D. guianense presentó estratificación en radios y parénquima axial, ratificando lo expuesto por Barghoorn et al. (1967), la especie P. copaifera registró fibras estratificadas, información que no ha sido reportada en estudios preliminares.

Las especies estudiadas se caracterizaron por la ausencia de elementos secretores; sin embargo, en $B$. floribunda se observaron células oleíferas y mucilaginosas asociadas al parénquima axial ratificando lo expuesto por Detienne \& Jacquet (1983); la especie C. officinalis presentó canales intercelulares axiales dispuestos en bandas tangenciales, confirmando la información reportada por León y Espinoza(2001), Baretta-Kuipers (1981), Detienne et al. (1982), Detienne \& Welle (1989), Miller \& Detienne (2001) y Gasson et al. (2003) citados en Melandri \& Espinoza (2009). Marcati et al. (2001) estudiaron las características estructurales de los canales intercelulares en otras especies de este género Copaifera langsdorffii Desf. Asi mismo, M. angustifolium y P. copaifera registraron canales intercelulares axiales difusos.

Otro carácter predominante fue la presencia de cristales prismáticos localizados predominantemente en células de parénquima axial. Las especies C. martiana, H. vageleri y M.angustifolium reportaron cristales en células radiales. La totalidad de las especies con cristales presentaron un cristal por célula o cámara. Por último, se encontraron cuerpos de sílice en forma de partículas en tres especies: C. glaberrima y T. polyphylla, en células radiales ratificando lo expuesto por Espinoza \& Melandri (2006), y en Dialium guianense en células de parénquima axial, como lo expusie- ron en sus estudios Kribs (1968), Nardi \& Edlmann (1992).

\section{CARACTERIZACIÓN CUANTITATIVA}

La anatomía cuantitativa aplicada a los elementos vasales ofrece información básica para conocer la forma como las condiciones ambientales influyen en la estructura anatómica de la madera. La mayoría de especies $(57,1 \%)$ se caracterizaron por tener vasos de diámetro grande, mientras que 5 especies presentaron una transición de grande a muy grande. Únicamente las especies Peltogyne pubescens y Peltogyne paniculata reportaron vasos de diámetro mediano y mediano a grande respectivamente mientras que K. malaccensis y T. polyphylla presentaron vasos de diámetro muy grande. En cuanto a la frecuencia de los poros, se tiene como categoría común: "muy poco frecuentes", aunque también se presentó un número considerable de especies, (38,1\%), con poros poco frecuentes. Tan solo $P$. pubescens reportó poros moderadamente frecuentes. Las especies presentan segmentos vasales de longitud media a baja. Predominó la presencia de punteaduras intervasculares pequeñas a medianas, mientras que en $K$. malaccensis y T. polyphylla se observaron punteaduras grandes.

Las especies estudiadas presentaron fibras de longitud media, excepto C. glaberrima que presentó fibras de longitud media a larga.

Los radios presentaron variaciones significativas en sus características cuantitativas. Predominó la ocurrencia de especies con radios compuestos de una a tres células de ancho. Sin embargo, la especie E. purpurea reportó radios hasta con seis células de ancho. En cuanto a la frecuencia, la mayoría de la especies $(81 \%)$ presentaron radios numerosos, siendo M. angustifolium, O. bernalii, P. paniculata y $S$. odoratissimum, las que presentaron radios muy numerosos. La mayoría de las especies presentan radios bajos y sólo E. purpurea, $P$. paniculata y $P$. copaifera presentaron radios con transición de bajos a altos. 


\section{ANÁLISIS DE SIMILITUD}

En la figura 8, se muestra el gráfico de conglomerados construido en base a las características microscópicas; se analizaron las similitudes existentes entre las especies al tratarse de características diagnósticas más específicas. Con este gráfico se puede deducir que las tres especies pertenecientes al género Peltogyne: P. paniculata, P. parvifolia y $P$. pubescens, muestran similitud a nivel microscópico, especialmente $P$. paniculata y $P$. parvifolia. Lo mismo ocurre con H. courbaril e H. oblongifolia, las cuales presentan un índice de similitud cercano a 0,8 . Estas similitudes ratifican las semejanzas dentro de los grupos taxonómicos e igualmente demuestran las diferencias que se pueden presentar dentro del mismo grupo, debido al grado de relación de los factores ambientales con la estructura anatómica de la madera. Por otra parte, M. oleifera y M. megistosperma, presentan un índice cercano a 0,8 lo cual indica que son muy similares entre sí, confirmando así, la sinonimia planteada por Schery 1951, D’Arcy 1987 y Ulloa et al. 2001 citados en Tropicos.org.

Las demás especies, aunque son únicas representantes de su género, permiten hacer otros análisis interesantes: P. copaifera se encuentra relacionada anatómicamente con E. purpurea, y H. vageleri con M. angustifolium; así mismo, C. glaberrima y C. officinalis presentan similitudes con las especies del género Hymenaea. Las demás especies son diferentes entre sí reportando índices inferiores a 0.65 , mientras que E. guineense y B. floribunda tienen cierto grado de similitud. Es importante observar que las especies T. polyphylla y S. odoratissimum, presentan un grado de similitud que se evidencia en el gráfico de conglomerados, estas dos especies,

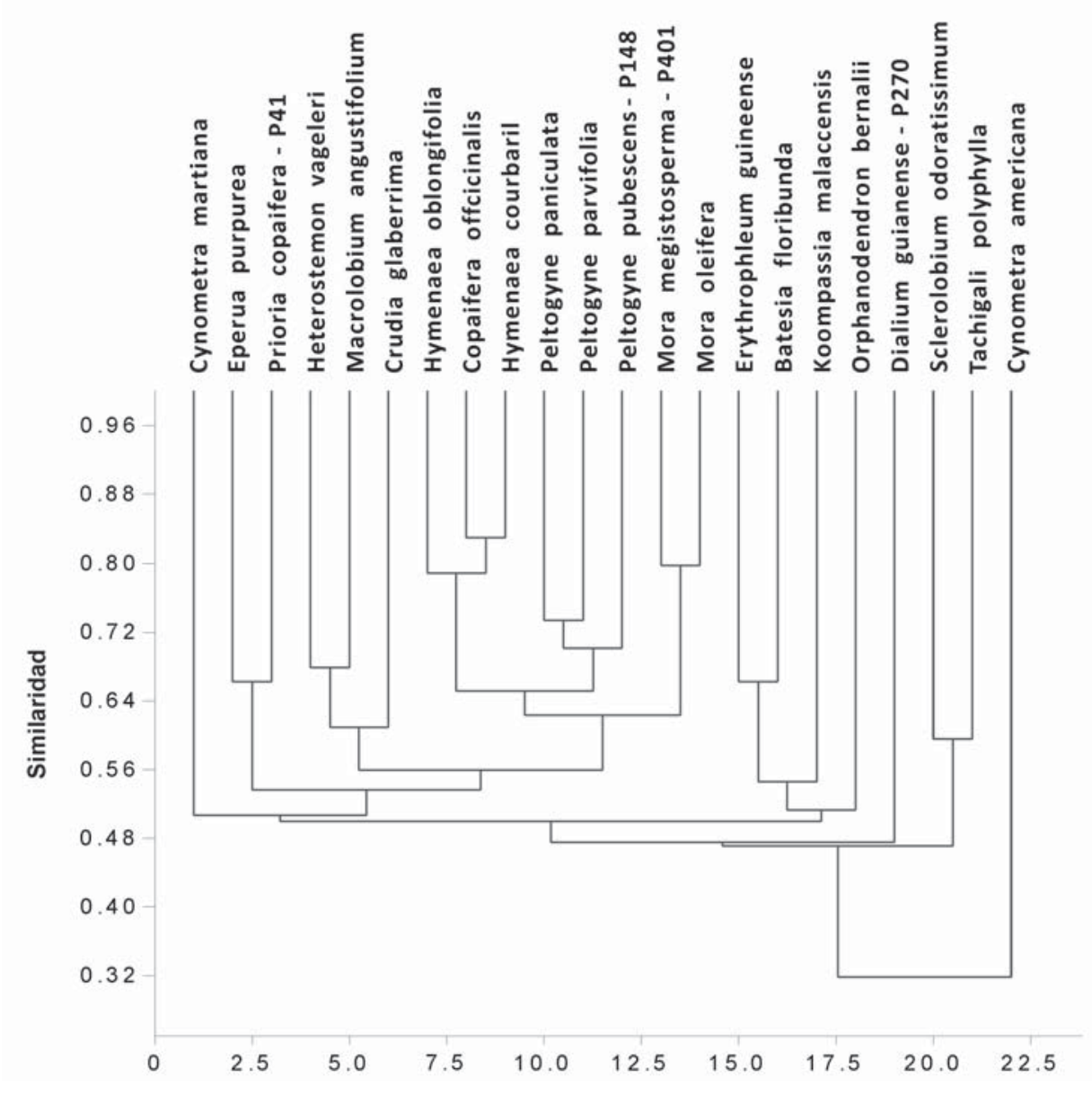

Figura 8. Gráfico de conglomerados basado en características microscópicas 
constituyen el inicio de las relaciones de similitud para la familia Caesalpiniaceae.

\section{CONCLUSIONES}

Las especies estudiadas las siguientes características anatómicas comunes, determinantes para la familia Caesalpiniaceae: diferenciación de anillos de crecimiento, porosidad difusa, poros solitarios sin patrón de disposición, platinas de perforación simple, punteaduras intervasculares areoladas alternas y ornadas, poros con contenidos gomosos, fibras no septadas con punteaduras simples o con reborde reducido, parénquima paratraqueal, radios de una a tres células de ancho, compuestos principalmente por células procumbentes. Por su parte, las características cuantitativas más comunes fueron vasos de diámetro grande y segmento vasal de longitud media, poros muy poco frecuentes, punteaduras pequeñas, fibras de longitud media y radios numerosos y bajos.

Se encontraron, igualmente, características restringidas a algunas especies y que pueden representar un valor de diagnóstico importante en la identificación a nivel de especie o género y para su diferenciación con otras especies de la familia Caesalpiniaceae. Tal es el caso de anillos de crecimiento indistinguibles en $B$. floribunda, $C$. martiana, E. guineense y $S$. odoratissimum y anillos definidos por disposición más compacta de las bandas de parénquima en la madera tardía en D. guianense, o distinción por banda de madera tardía de coloración más oscura en T. polyphylla.

Las especies E. purpurea, S. odoratissimum y $T$. polyphylla presentaron poros en disposición radial oblicua. Las especies $P$. pubescens, K. malaccensis y T. polyphylla, presentaron poros de diámetro mediano a grandes y vasos con punteaduras grandes. Las especies $C$. martiana, $H$. vageleri y M. oleífera presentaron fibras con punteaduras con reborde.

Las especies $M$. angustifolium y $T$. polyphylla, presentaron parénquima paratraqueal escaso, mientras la especie $D$. guianense desarrolla parénquima reticulado, y la especie E. guineense muestra un parénquima axial apotraqueal difuso en agregados. Así mismo, se encontraron estructuras estratificadas en las especies D. guianense y $P$. copaifera con fibras estratificadas. La especie B. floribunda contiene células oleíferas y mucilaginosas asociadas al parénquima axial. La especie C. officinalis desarrollo canales intercelulares axiales en bandas tangenciales. Por último, se encontraron cuerpos de sílice en C. glaberrima, $D$. guianense y $T$. polyphylla.

Las características anatómicas del tejido xilemático de las especies estudiadas también permitieron constatar las relaciones taxonómicas de algunas especies de la familia Caesalpiniaceae. De esta manera se comprobó que las especies M. Megistosperma y M. oleífera; P. pubescens y P. paniculata; y S. odoratissimum y T. polyphylla, se encuentran relacionadas taxonómicamente, ratificando la aplicación de la anatomía de maderas a los estudios taxonómicos, principalmente en aquellas situaciones en las que no se cuenta con muestra botánica.

Se incorporó el estudio de la estructura anatómica de la especie $O$. bernalii, de la cual no se registran estudios anatómicos a pesar de ser una especie de amplio uso en la región del Pacifico y Medio Atrato antioqueño en Colombia.

En síntesis, este trabajo se consolidó como un importante aporte al conocimiento de la familia Caesalpiniaceae en el campo de la anatomía de la madera y sus aplicaciones, al describir especies que cuentan con poca información y de aquellas de las cuales no se refieren estudios anatómicos.

\section{AGRADECIMIENTOS}

Los autores expresamos nuestros agradecimientos al Centro de Investigaciones y Desarrollo Científico de la Universidad Distrital Francisco José de Caldas, al Grupo de investigación "Uso y Conservación de la Diversidad Forestal, y al equipo técnico de anatomía de la madera del Laboratorio de 
Tecnología de Maderas de la Universidad Distrital, por sus invaluables aportes al desarrollo de la presente investigación.

\section{REFERENCIAS BIBLIOGRAFICAS}

Barghoorn, A., M. Reyes, L. Rojas, D. Cabrera \& P. Alvarado. 1967. Estudio orientativo de algunas propiedades anatómicas y físico mecánicas de 41 especies maderables de la región Carare - Opón. Instituto de Investigaciones y Proyectos Forestales y Madereros de la Universidad Distrital Francisco José de Caldas. Bogotá.

Carlquist, S. 2001 Comparative Wood anatomy, Sistematic, ecological and evolutionary aspects of dicotyledon Wood. Springer Verlag. Berlin y Heidelberg. 448 pp.

Cárdenas, D. \& N. Salinas (eds.). 2007. Libro rojo de plantas de Colombia. Especies maderables amenazadas I parte. Instituto Amazónico de Investigaciones Científicas, SINCHI. Ministerio de Ambiente, Vivienda y Desarrollo Territorial. Bogotá. 232 p.

Chase, M. 2003. An update of the Angiosperm Phylogeny Group classification for the orders and families of flowering plants: APG II. Botanical Journal of the Linnean Society 141: 399-436.

Cronquist, A. \&1981. An Integrated System of Classification of Flowering Plants. Columbia University Press. New York. 1262 p.

Detienne, P. \& P. Jacquet. 1983. Atlas d'identification des bois de l'amazonie et des regions voiseines. Centre Technique Forestier Tropical, Inside Wood .Nogent s/Marne. 640 pp.

Díaz de Méndez, H. 1963. Resultado del estudio de 47 maderas de la Guayana Venezolana. Universidad de los Andes, Facultad de Ciencias Forestales. Mérida.

Espinoza, N. \& J. Melandri. 2006. Wood anatomy of the tribe Caesalpinieae (LEGUMINO-
SAE, CAESALPINIOIDEAE), in Venezuela. IAWA Journal 27(1) 99-114.

García, L., A. \& Guindeo, C. Peraza \& P. de Palacios. 2003. La madera y su anatomía: Anomalías y defectos, estructura microscópica de coníferas y frondosas. Asociación de Investigación Técnica de las Industrias de la Madera y Corcho. Editorial Mundi - Prensa. Madrid. 327 p.

Gassen, P. 1999. Wood anatomy of the trme dipterygeae with comments on related papilionoid and caesalpinioid leguminosae. Royal Botanic Gardens, Kew, Riehmond. Snrrey, TW9 3AB, United Kingdom. LAWA Journal 20 (4): 441455 .

Höhn, A. 1999. Wood anatomy of selected West African species of Caesalpiniodeae and Mimosoideae (Leguminosae): A comparative study. IAWA Journal 20: 115-146

IAWA Committee. 1989. List of Microscopic Features for Hardwood Identification. International Association of Wood Anatomists. IAWA Bulletin 10.

Inside Wood. 2004 (en adelante). [Base de datos en línea]. [Consultado el 02 de Agosto de 2010]. Disponible en $<\mathrm{http}$ ://insidewood.lib. ncsu.edu/search>.

JUNAC. 1981. Descripción general y anatómica de 105 maderas del grupo andino. Junta del Acuerdo de Cartagena. Lima.

Kribs, D. 1968. Commercial foreign woods on the American market. Dover Publications. New York, United States. Recuperado el 06 de Agosto de 2010 de la base de datos Inside Wood. $<$ http://insidewood.lib.ncsu.edu/search $>$.

León, W. \& N. Espinoza de P. 2001. Anatomía de la Madera. Universidad de los Andes, Consejo de Publicaciones. Mérida. 397 p.

López, R \& I. Montero. 2005. Manual de identificación de especies forestales en bosques naturales con manejo certificable por comunida- 
des. Instituto Amazónico de Investigaciones Científicas, SINCHI. Bogotá.

Mahecha, G. \& R. Echeverri. 1983. Árboles del Valle del Cauca. Litografía Arco. Bogotá. $208 \mathrm{p}$.

Mainieri , C. \& J. Peres. 1989. Fichas de características das madeiras brasileiras. 2 ed. Sao Paulo: Instituto de Pesquisas Tecnológicas, Divisao de Madeiras 307-308. p

Marcati ,C. \& V. Angyalossy-Alfonso. 2001 Anatomia comparada do lenho de Copaifera langsdorffii Desf. (Leguminosae-Caesalpinoideae) de floresta e cerradão. Revta. brasil. Bot. 24(3): 311-320.

Melandri \& Espinoza 2009 Wood anatomy of tribe Detarieae and comparison with tribe Caesalpinieae (Leguminosae, Caesalpinioideae) in Venezuela. \&Revista Biologia Tropical 57 (1-2): 303-319

Miller, R. \& P. Détienne. 2001. Major timber trees of Guyana. Wood anatomy. Tropenbos International, Wageningen. Recuperado el 11 de Agosto de 2010 de la base de datos Inside Wood. <http://insidewood.lib.ncsu.edu/ search>.

Miller, R. 2007. Fluorescent woods of the world, pp.: 271-305. En: J.H. Flynn, Jr. (ed.). A Guide to the More Useful Woods of the World. Forest Products Society, Madison, WI

Moya, R., M. Gómez \&J. Rivero. 2007. Clave de identificación macroscópica para 22 especies maderables de Bolivia. Revista Forestal Venezolana 51 (2)179-193.

Nardi, R. \& M. Edlmann. 1992. Legnami tropicali importati in Italia: anatomia e identifi- cazione. Vol. II. América Latina. \&CNR, Firenze. Recuperado el 11 de Agosto de la base de datos Inside Wood. <http://insidewood.lib. ncsu.edu/search $>$.

PROEXPO. 1970. Maderas Colombianas. Fondo de promoción de exportaciones. Bogotá. $117 \mathrm{p}$.

Quirk, T. 1983. Data for a computer-assisted wood identification system. Commercial Legumes of tropical Asia and Australia. \&IAWA Bull. 4: 118-130.

Richter, H. \& M. Dallwitz. 2000. Commercial timbers: descriptions, illustrations, identification, and information retrieval. In English, French, German, Portuguese, and Spanish. Disponible en $<$ http://delta-intkey.com $>$.

Rodríguez, L. 1988. Caracterización anatómica de las maderas latifoliadas y claves macro y microscópica para la identificación de 120 especies. Tesis de grado Ingeniería Forestal. Universidad Distrital Francisco José De Caldas. Bogotá.

Soerianegara, I. \& R. Lemmens. 1993. Plant Resources of South-East Asia. No. 5 (1). Timber trees: major commercial timbers. Pudoc Scientific Publishers. Wageningen. 610 p.

Stevens, P. 2001. Angiosperm Phylogeny Website. Version 9, June 2008 [Base de datos en línea]. [Consultado el 18 abril de 2009]. Disponible en $<$ http://www.mobot.org/MOBOT/ research/APweb/>.

Vásquez, A. \& Ramírez, A. 2005. Maderas comerciales en el Valle de Aburrá. Área Metropolitana del Valle de Aburrá, subdirección ambiental. Medellín. 


\section{ANEXO}

\section{CLAVES DICOTÓMICAS PARA IDENTIFICACIÓN AL NIVEL DE ESPECIE}

Clave de identificación macroscópica

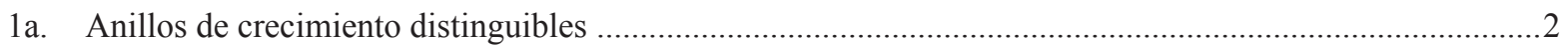

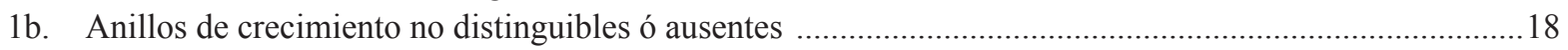

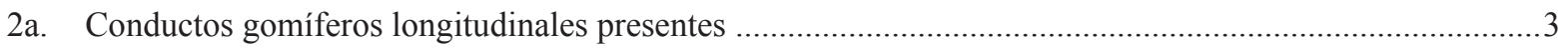

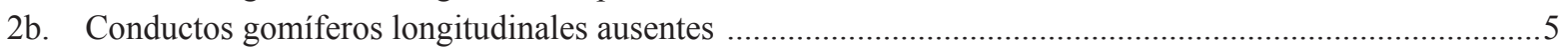

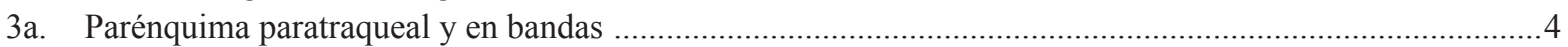

3b. Parénquima apotraqueal, paratraqueal y en bandas ....................................................... Copaifera officinalis

4a. Parénquima paratraqueal escaso y vasicéntrico ................................................ Macrolobium angustifolium

4b. Parénquima vasicéntrico y aliforme .................................................................................Prioria copaifera

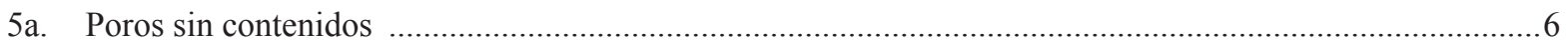

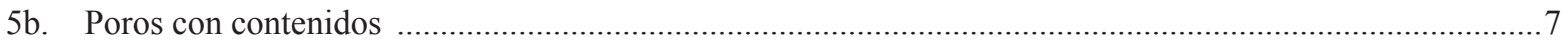

6a. Poros en patrón de disposición diagonal ...................................................................... Tachigali polyphylla

6b. Poros sin patrón de disposición ................................................................................ Heterostemon vageleri

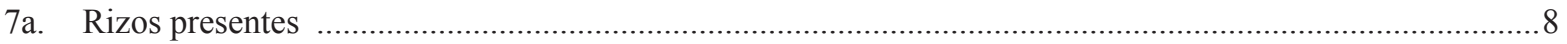

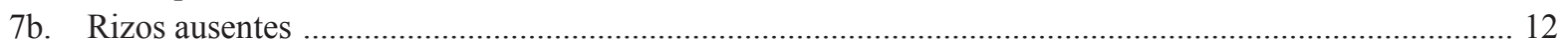

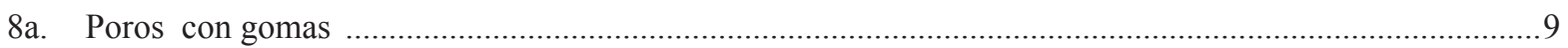

8b. Poros con depósitos de calcio y goma .................................................................. Orphanodendron bernalii

9a. Poros medianos (escasamente visible a simple vista) ....................................................................... 10

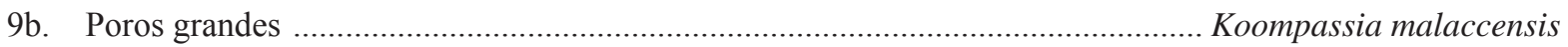

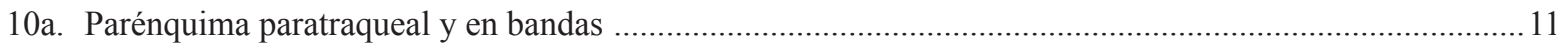

10b. Parénquima exclusivamente en bandas ........................................................................ Crudia glaberrima

11a. Parénquima en bandas reticulado ........................................................................... Dialium guianense

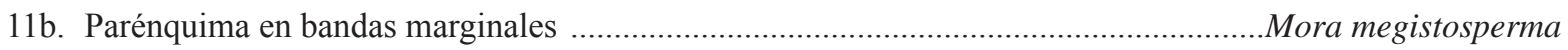

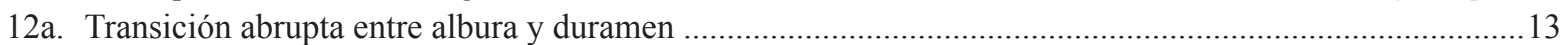

12b. Transición perceptible entre albura y duramen....................................................................... Mora oleifera

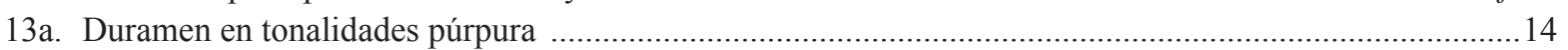

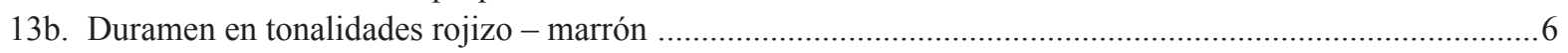

14a. Radios finos (visibles con lupa de 10X con dificultad) ........................................................................ 15

14b. Radios medianos (claramente visibles con lupa de 10X) ..............................................Peltogyne parvifolia

15a. Poros pequeños (difícilmente visibles con lupa de 10x)............................................Peltogyne paniculata

15b. Poros muy pequeños (no visibles con lupa de 10x) .................................................. Peltogyne pubescens

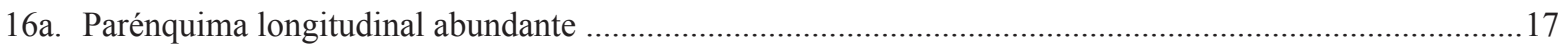

16b. Parénquima longitudinal escaso ................................................................................... Eperua purpurea

17a. Poros medianos (visibles a simple vista con alguna dificultad) ......................................Hymenaea courbaril

17b. Poros grandes (claramente visibles a simple vista) .................................................. Hymenaea oblongifolia

18a. Poros solitarios y múltiples radiales en similar proporción ...............................................................19

18b. Poros exclusivamente solitarios ( + del 90\% solitarios). .............................................. Batesia floribunda

19a. Radios medianos (claramente visibles con lupa de 10x) .......................................................................20

19b. Radios finos (visibles con lupa de 10x) .................................................................. Erythrophleum guineense

20a. Parénquima apotraqueal y en bandas ...................................................................... Cynometra martiana

20b. Parénquima paratraqueal ..........................................................................Sclerolobium odoratissimum 


\section{CLAVE DE IDENTIFICACIÓN MICROSCÓPICA}

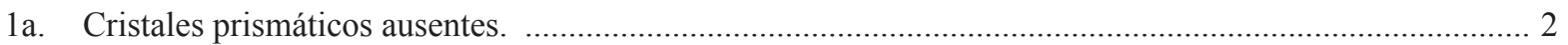

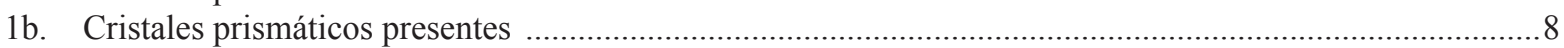

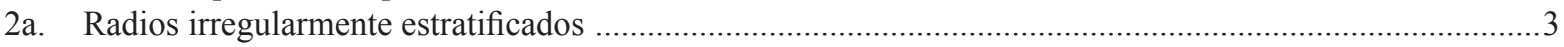

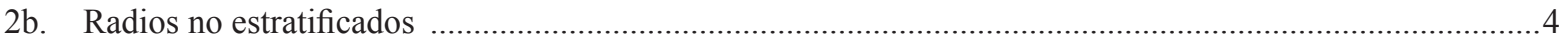

3a. Parénquima aliforme. ....................................................................................... Koompassia malaccensis

3b. Parénquima en bandas ....................................................................................... Orphanodendron bernalii

4a. Células oleíferas y mucilaginosas ausentes ................................................................................... 5

4b. Células oleíferas y mucilaginosas presentes ............................................................... Batesia floribunda

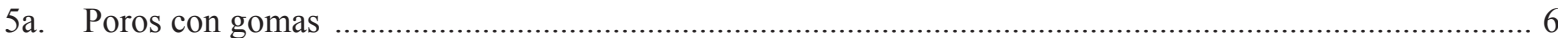

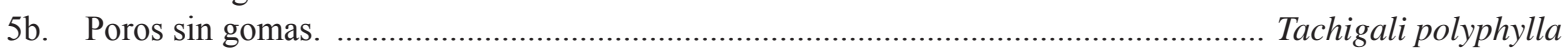

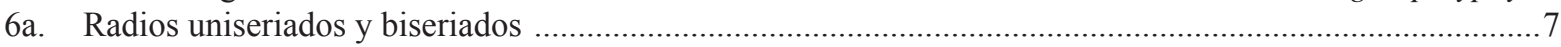

6b. Radios multiseriados (>4 células de ancho) .................................................................. Eperua purpurea

7a. Poros en patrón de disposición diagonal ..........................................................Sclerolobium odoratissimum

7b. Poros sin patrón de disposición definido ................................................................Erythrophleum guineense

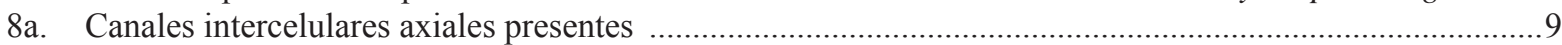

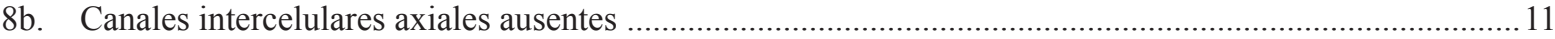

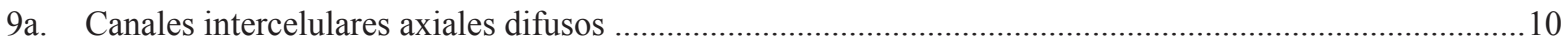

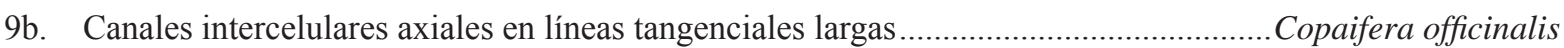

10a. Fibras estratificadas ....................................................................................................Prioria copaifera

10b. Fibras no estratificadas .............................................................................. Macrolobium angustifolium

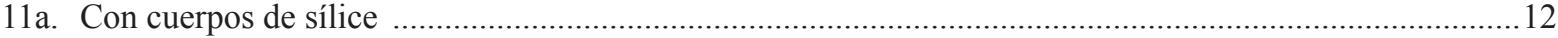

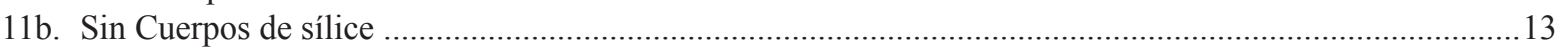

12a. Cuerpos de sílice presentes en células de parénquima axial ............................................. Dialium guineense

12b. Cuerpos de sílice presentes en células radiales ........................................................... Crudia glaberrima

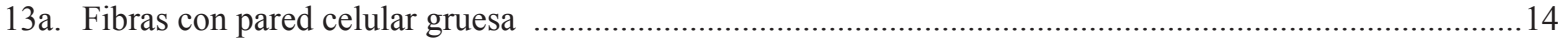

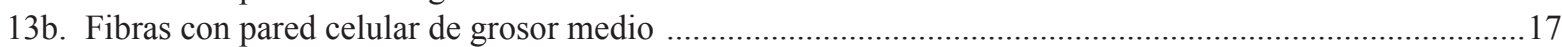

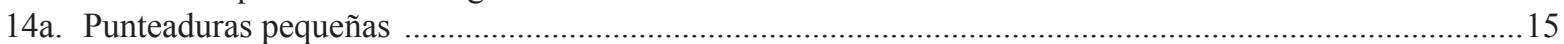

14b. Punteaduras medianas ..............................................................................................menaea courbaril

15 a. Cristales prismáticos presentes en células de parénquima axial .............................................................16

15b. Cristales prismáticos presentes en las células radiales ................................................ Cynometra martiana

16a. Radios uniseriados, biseriados, triseriados y multiseriados ..........................................Peltogyne paniculata

16b. Radios uniseriados, biseriados y triseriados ............................................................. Peltogyne pubescens

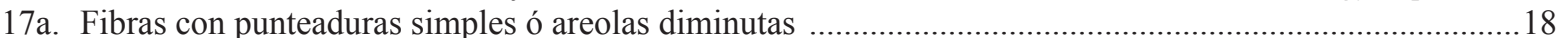

17b. Fibras con punteaduras areoladas distinguibles ...................................................... Heterostemon vageleri

18a. Parénquima en serie de 3-8 células ...........................................................................................................................19

18b. Parénquima en serie de $3-4$ células ..................................................................................................eltogyne parvifolia

19a. Poros muy poco frecuentes $\left(<=5\right.$ vasos $\left./ \mathrm{mm}^{2}\right)$......................................................................... Hymenaea oblongifolia

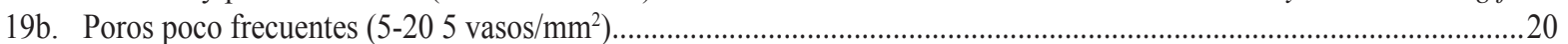

20a. Parénquima paratraqueal de ala ancha y corta, parénquima paratraqueal confluente................................. Mora oleífera

20b. Parénquima paratraqueal aliforme, parénquima en bandas finas y en bandas anchas.........................ora megistosperma 